${ }^{1}$ Singapore National Eye Center, Singapore Eye Research Institute, Singapore, Singapore

${ }^{2}$ Singapore Eye Research Institute, Singapore Singapore

${ }^{3}$ Duke-NUS Medical School, Singapore, Singapore

${ }^{4}$ Department of Ophthalmology, University Hospitals of Birmingham NHS Foundation Trust, Birmingham, UK

${ }^{5}$ Academic Unit of Ophthalmology, Institute of Inflammation and Ageing, University of Birmingham, Birmingham, UK

${ }^{6}$ National Institute for Health Research (NIHR) Biomedical Research Centre at Moorfields Eye Hospital NHS Foundation Trust and UCL Institute of Ophthalmology, London, UK

${ }^{7}$ Department of Ophthalmology, Queen's University of Belfast, Royal Victoria Hospital, Belfast, Northern Ireland

Correspondence: CMG Cheung, Singapore National Eye Center, Singapore Eye Research Institute, level 8, 11 Third Hospital Avenue, Singapore 168751, Singapore Tel: +65 6227 7255; Fax: +65 63793519 . E-mail: gemmy.cheung.c. m@singhealth.com.sg

Received: 18 April 2017 Accepted in revised form: 15 July 2017

Published online:

8 September 2017

\section{An overview of the clinical applications of optical coherence tomography angiography}

\begin{abstract}
Optical coherence tomography angiography (OCTA) has emerged as a novel, non-invasive imaging modality that allows the detailed study of flow within the vascular structures of the eye. Compared to conventional dye angiography, OCTA can produce more detailed, higher resolution images of the vasculature without the added risk of dye injection. In our review, we discuss the advantages and disadvantages of this new technology in comparison to conventional dye angiography. We provide an overview of the current OCTA technology available, compare the various commercial OCTA machines technical specifications and discuss some future

\section{Method}

This comprehensive literature review was performed based on a search of peer-reviewed published papers relevant to optical coherence tomography angiography (OCTA) according to our current knowledge, up to January 2017, available on the PubMed database. This review will highlight OCTA technology and software updates relevant to clinicians and discuss clinical approaches to the interpretation of OCTA keeping in mind its limitations and artefacts. We will then examine some current clinical applications of this technology and implications for future use.
\end{abstract} software improvements. An approach to the interpretation of OCTA images by correlating images to other multimodal imaging with attention to identifying potential artefacts will be outlined and may be useful to ophthalmologists, particularly those who are currently still unfamiliar with this new technology. This review is based on a search of peer-reviewed published papers relevant to OCTA according to our current knowledge, up to January 2017, available on the PubMed database. Currently, many of the published studies have focused on OCTA imaging of the retina, in particular, the use of OCTA in the diagnosis and management of common retinal diseases such as age-related macular degeneration and retinal vascular diseases. In addition, we describe clinical applications for OCTA imaging in inflammatory diseases, optic nerve diseases and anterior segment diseases. This review is based on both the current literature and the clinical experience of our individual authors, with an emphasis on the clinical applications of this imaging technology.

Eye (2018) 32, 262-286; doi:10.1038/eye.2017.181; published online 8 September 2017
ACS Tan ${ }^{1,2,3}$, GS Tan ${ }^{1,2,3}$, AK Denniston 4,5,6, PA Keane ${ }^{6}$, M Ang $^{1,2,3}$, D Milea ${ }^{1,2,3}$, U Chakravarthy ${ }^{7}$ and CMG Cheung ${ }^{1,2,3}$

\section{Overview of technology}

OCTA is a novel imaging modality that allows the detailed three-dimensional study of blood flow within the vascular structures of the eye without the need to intravenously administer fluorescent dyes. ${ }^{1,2}$ OCTA technology is based on detecting differences in amplitude, intensity or phase variance between sequential B-scans taken at the same location of the retina. ${ }^{1}$ Briefly, a series of B-scans are collected at the same transverse location and registered. The degree of decorrelation in signal is then calculated, which enables visualisation of only the moving part, assumed to be due to movement of cells within the blood stream and thus blood flow. The above procedure is then repeated for different Y-position in the retina to achieve the threedimensional data set, from which proprietary algorithms such as split-spectrum amplitudedecorrelation angiography, optical microangiography and OCT angiography ratio analysis are used to reconstruct en face angiograms (Figure 1).

OCTA offers several advantages compared to conventional angiography. The non-invasive 


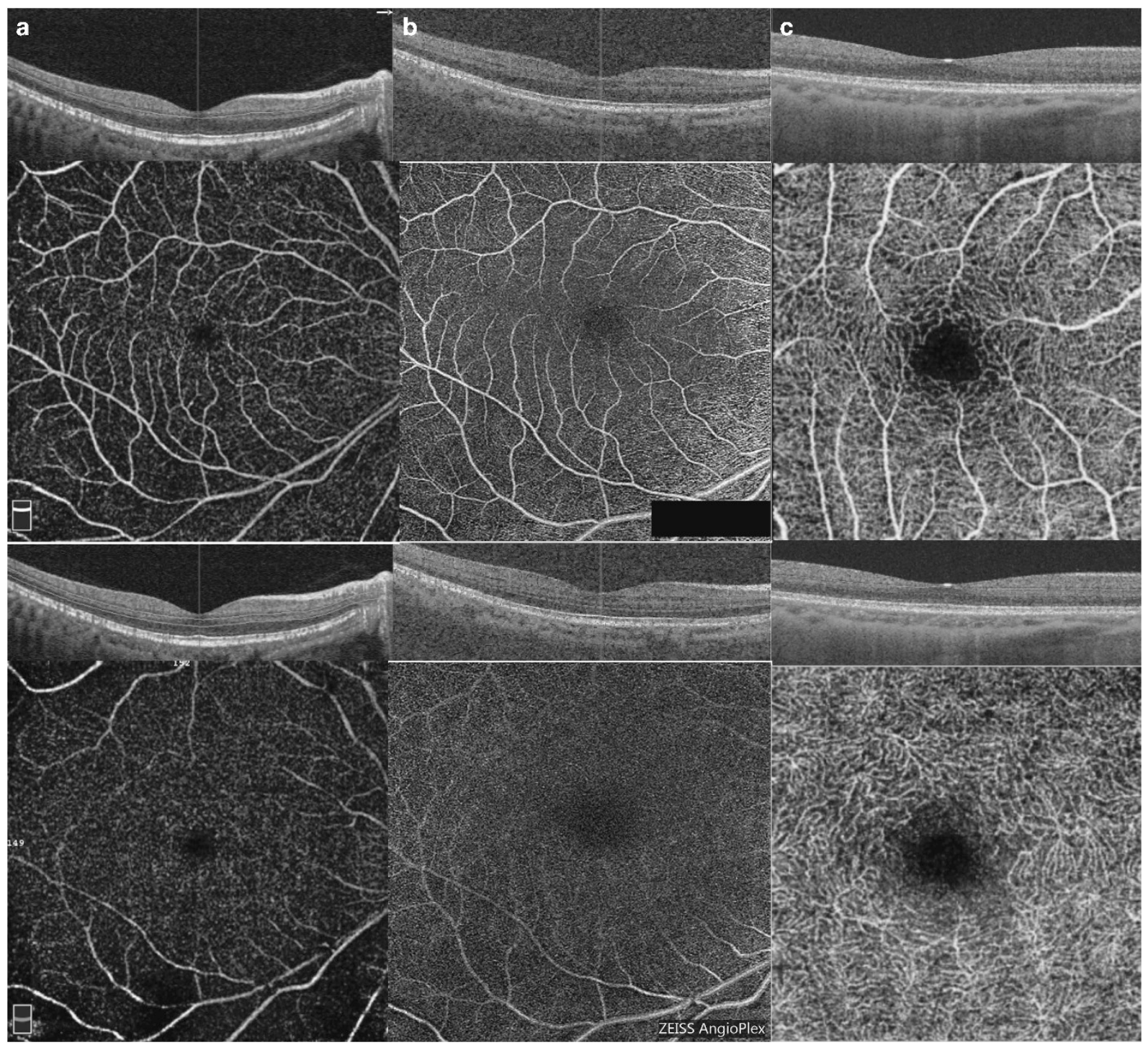

Figure 1 OCTA of a single normal eye showing variations in the scan area and algorithms. Cross-sectional OCTA images of the superficial vascular plexus segmentation (first row) and deep vascular plexus (third row). En face OCTA images of the superficial vascular plexus segmentation (second row) and deep vascular plexus (bottom row). (a) An $8 \times 8 \mathrm{~mm}$ scan taken with the AngioVue (Fremont, CA, USA) RTVue XR Avanti processed with the SADA algorithm; (b) A $6 \times 6 \mathrm{~mm}$ scan taken with the Angioplex (Dublin, CA, USA) CIRRUS HD-OCT Model 5000 processed with the optical microangiography algorithm; (c) A $3 \times 3$ mm scan taken with DRI-OCT Triton (Tokyo, Japan) swept source OCT processed with the OCTA-RA algorithm. On the automated segmentation of the deep vascular plexus of both the Angiovue and Angioplex, some projection artefact from the superficial layer is observed. A full color version of this figure is available at the Eye journal online.

nature and fast acquisition time allows this test to be repeated frequently and avoids the potential risks associated with intravenous dye injection (Table 1). In addition, high-resolution details of the vasculature and depth-resolved analysis, in which the flow within a specific axial location of the retinal or choroid can be analysed (Table 1). The absence of functional information such as the severity of exudation and filling speed as well as stereoscopic viewing and wide-field functions (Table 1) are other disadvantages of OCTA compared to conventional angiography.
Automated, objective quantitative measures (angio-analytics) of flow have been incorporated into many OCTA platforms (Table 2). ${ }^{3}$ These software developments are still in their infancy and need to be tested for intra- and inter-platform reliability and repeatability, in both normal and diseased eyes. Some instruments offer a function to 'register' two different visits by aligning features on the en face images. This function is useful for assessing treatment response and disease progression (Table 2). ${ }^{4-7}$ 
Table 1 Comparison of OCTA versus conventional angiography such as fundus FA and ICGA

\begin{tabular}{|c|c|c|}
\hline OCTA & $F F A$ & ICGA \\
\hline New technology not validated & Well-validated technology & Well-validated technology \\
\hline $\begin{array}{l}\text { Correlation to multimodal imaging and } \\
\text { histology }\end{array}$ & $\begin{array}{l}\text { Correlation to multimodal imaging and } \\
\text { histology }\end{array}$ & \\
\hline Non-invasive, no need for dye & Invasive, need for dye risk of anaphylaxis & $\begin{array}{l}\text { Invasive, need for dye risk of } \\
\text { anaphylaxis }\end{array}$ \\
\hline Rapid acquisition time & Time-consuming to perform & Time-consuming to perform \\
\hline Interpretation may require more time & Image viewing may be faster & Image viewing may be faster \\
\hline $\begin{array}{l}\text { Provides depth information of both retinal } \\
\text { and choroidal vasculature }\end{array}$ & No information about individual layers & No information about individual layers \\
\hline Able to segment various layers & Retina imaged in entirety & Choroid imaged in entirety \\
\hline Able to image through blood & Blockage from blood & Able to penetrate blood \\
\hline Artefacts may hamper interpretation & Less artefact & Less artefact \\
\hline Detection of flow but not leakage & Detection of leakage and activity & Detection of leakage and activity \\
\hline $\begin{array}{l}\text { High resolution down to capillaries in the } \\
\text { retina }\end{array}$ & $\begin{array}{l}\text { Lower resolution, able to image large retinal } \\
\text { vessels but not capillaries }\end{array}$ & $\begin{array}{l}\text { Able to image large choroidal vessels but } \\
\text { not choriocapillaries }\end{array}$ \\
\hline Small field of view & Wide-field option available & Wide-field option available \\
\hline No stereoscopic function & Stereoscopic option & Stereoscopic option \\
\hline No dynamic video function & Video function available & Video function available \\
\hline
\end{tabular}

\section{OCTA interpretation and potential artefacts}

In our experience, high-quality image acquisition for each OCTA platform has a learning curve; hence good technical support is essential and poor quality images should be identified. Instrument-related factors that may affect image quality include differences in acquisition time. Patient-related factors include age, ability to cooperate and maintain fixation, and the presence media opacity.

Similar to image acquisition, OCTA interpretation by the clinician, also has a learning curve. The user interfaces of most of the current OCTA platforms vary; however, the basic components are similar. An approach to OCTA interpretation is outlined below (Figure 2).

\section{Assess the scan quality}

This should include assessment of the scan centration, resolution and signal strength. Signal strength may be affected by patient co-operation, fixation or medial opacity.

\section{Identify the layer and the area of interest}

Through a detailed clinical exam and examination of the structural OCT, the clinician should be able to determine at which layer (retinal versus choroidal) the pathology lies and the area of interest to be scanned by the OCTA, keeping in mind the various scan area options available on the various OCTA platforms. If the pathology is not around the macula, an OCTA scan decentred from the fovea may be necessary.

\section{Examine the cross-sectional OCTA for abnormal flow}

Most OCTA platforms represent the detected flow signals by superimposing them onto a structural B-scan OCT images in a coloured overlay to derive a cross-sectional OCTA image (Figure 1). Scrolling through the crosssectional OCTA images to look for abnormal flow in the layer and area of interest will help locate the corresponding area on the en face OCTA to focus on.

\section{Choose the preset segmentation pattern that best captures the area of abnormal flow}

All the commercially available OCTA instruments are built with automated segmentation (Table 2). If no particular segmentation pattern is able to accurately capture the area of abnormal flow, (for example, for studying large choroidal vessels or pre-retinal neovascularisation), customised segmentations patterns may be necessary to obtain an optimised en face OCTA image.

\section{Manual manipulation of the segmentation to optimise the en face OCTA image}

The exact depth and thickness of the preset segment varies according to individual instrument. Further manual adjustment of the lower and the upper boundaries of various segmentation patterns will allow the en face OCTA image to be easily tailored towards the clinical question. In some pathological cases, where the anatomy is severely disrupted, automated segmentation may not be accurate and the adjustment of the contour on each of the individual B-scan segmentation lines may be 


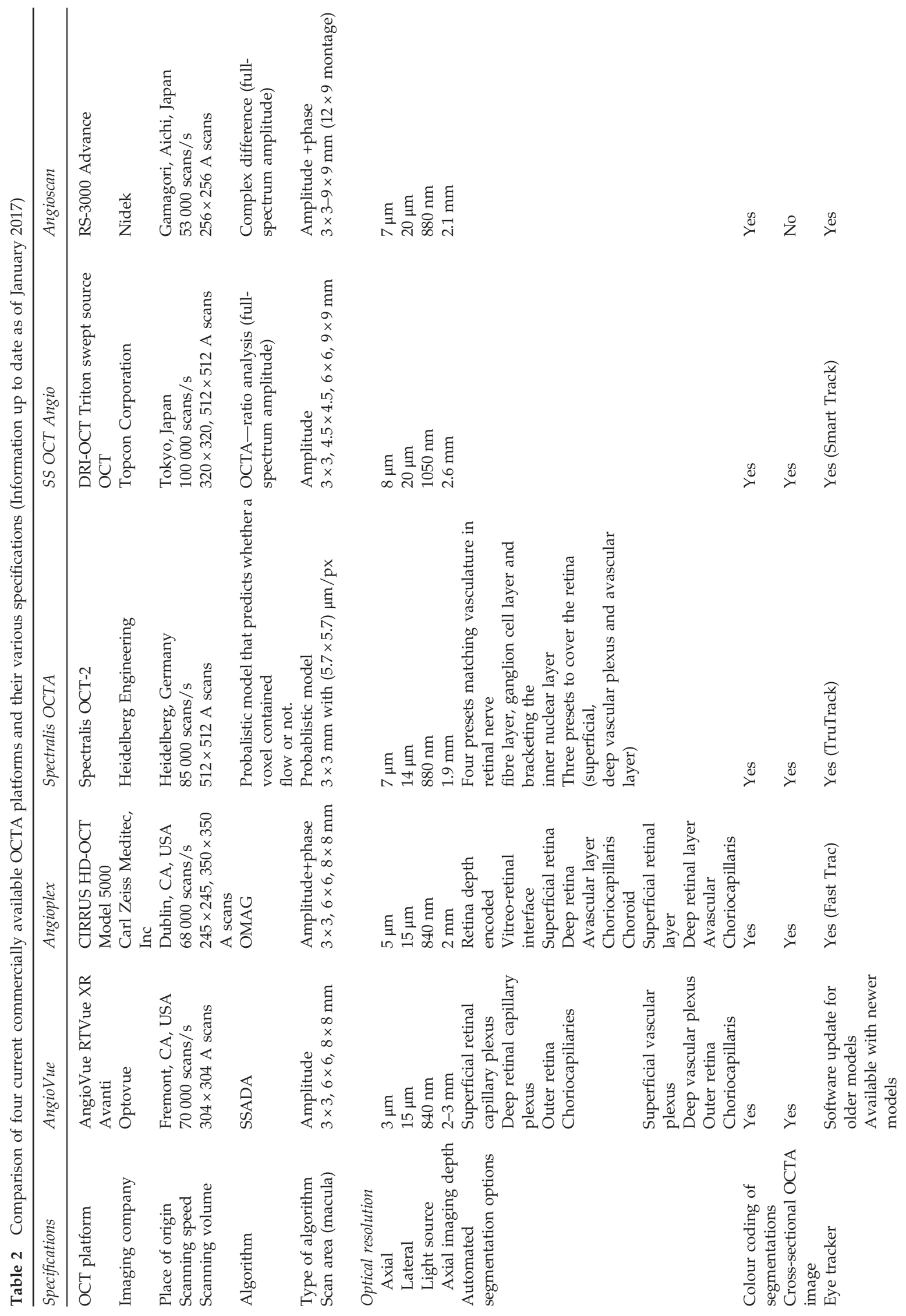




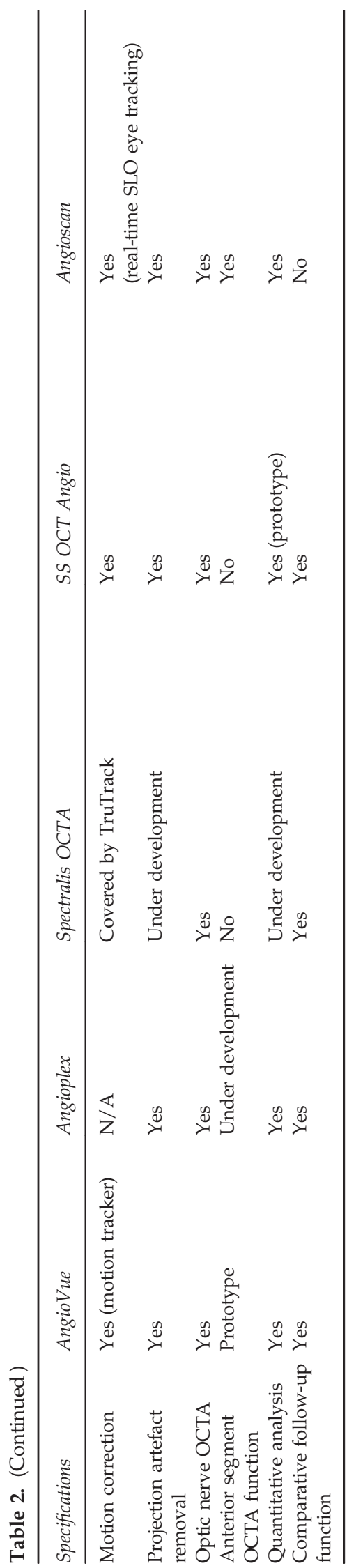

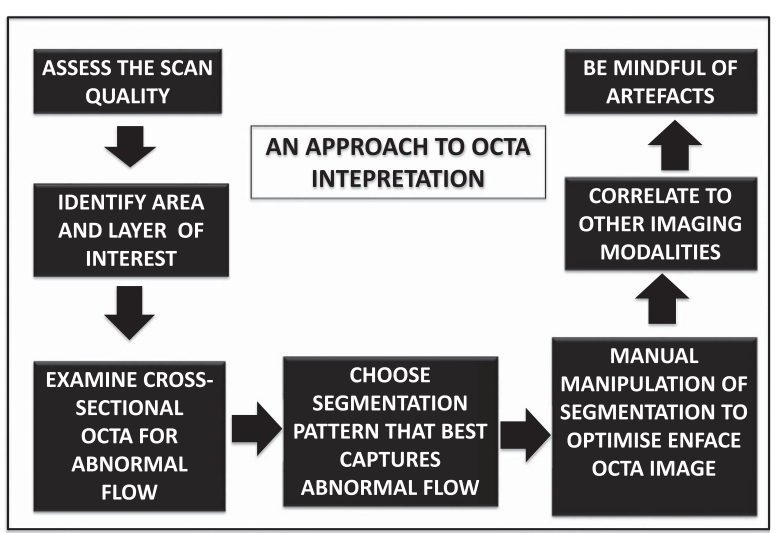

Figure 2 A summary of an approach to OCTA interpretation.

required to optimise the en face OCTA image (Figure 3d). This manual adjustment of the contour is both time and labour intensive. However upgrades in the software, such as auto-propagation of manual changes have shortened the time to perform such adjustments may improve this function.

\section{Correlate to other imaging modalities}

OCTA is a new technology, which has yet to be validated; hence, interpretation should be done with caution and in equivocal cases correlation with more conventional modalities such as fundus fluorescein angiography (FA) or indocyanine green angiography (ICGA).

\section{Be mindful of artefacts}

Understanding the types and sources of artefacts is important during the interpretation of OCTA (Figure 3). 8,9 Motion artefacts caused by blinking results in dark lines, while motion artefacts due to saccadic eye movements or bulk movements usually appear as horizontal white lines and can be minimised with a few strategies such as orthogonal image registration (Figure 3a), ${ }^{9,10}$ the incorporation of an eye-tracker or a combination of tracking-assisted scanning integrated with motion correction technology (Table 2). ${ }^{11}$ In theory, less motion artefact should occur with a more sensitive the eyetracker; however, a highly sensitive eye tracker may increase the acquisition time and make imaging challenging.

As previously stated, segmentation errors are common in pathology, in which the retinal architecture is altered. Inaccurate segmentation may result in dark areas on the en face OCTA image (Figure 3d). Scrolling through the structural OCT volume scan will allow identification of areas with inaccurate segmentation. Manual adjustment 


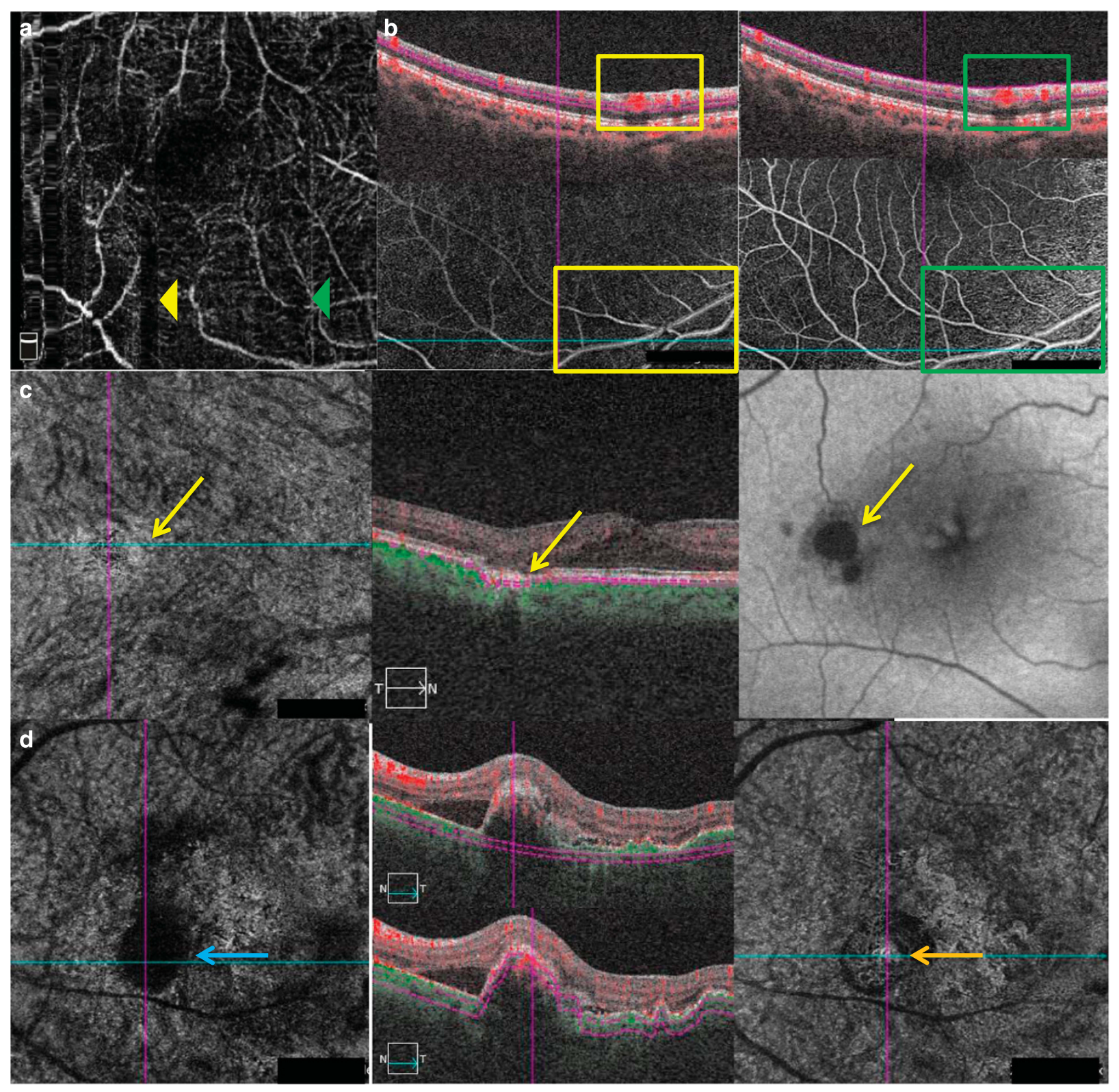

Figure 3 Examples of common artefacts seen on OCTA. (a) Motion artefact seen by black vertical lines caused by blinking (yellow arrowhead) and eye movements (green arrowhead). (b) An example of a projection artefact (yellow boxes) of the superficial vessels seen in the deep vascular plexus segmentation. Comparing the deep vascular plexus segmentation, all the projection artefact seen can be accounted for by the more superficial vessels (green boxes). (c) Unmasking artefact seen as an area of high flow (middle of crosshairs) on en face OCTA (left), cross-sectional OCTA (middle) showed a focal area of atrophy with underlying hyper-transmission of the signal (yellow arrow). Area of high flow on en face OCTA can be accounted for by an area of atrophy causing the underlying choroidal vessels to be seen as an area of unmasking artefact. This is confirmed by the corresponding area of hypo-autofluorescence seen on fundus autofluorescence (right). (d) En face OCTA (left) corresponding to cross-sectional OCTA (middle top) showing a straight segmentation line that does not capture polyps (blue arrow) seen at the peak of the pigment epithelial detachment. Alternatively, when using the RPE fit segmentation the area of polyps (orange arrow) are then seen on en face OCTA

should be performed before interpretation of the final en face OCTA.

Projection artefacts occur in highly reflective layers of the retina such as the retinal pigment epithelium (RPE) (Figure 3). $8,9,12,13$ When superficial retinal flow signals
(Figure 3b-green box) are reflected off the deeper layers, they will be detected as decorrelation signals that possess the same character as overlying blood vessels. (Figure $3 \mathrm{~b}$ -yellow box). ${ }^{12}$ As a result, flow may be inaccurately interpreted to be present within a deeper structure, when 
the flow signals actually originated from the more superficial layers. Most OCTA platforms possess in-built software to mask the projection artefacts in the outer retinal layers. ${ }^{14}$ However, this software has limitations, as projection artefacts may occur in various other layers, especially in hyperreflective pathological structures such as hard exudates and subretinal fibrosis. ${ }^{14,15} \mathrm{~A}$ useful way to ascertain whether the flow signal seen is due to projection artefact is by examining the cross-sectional OCTA (or Angio B scan), in which the linear signals can be traced to flow within a more superficial layer (Figure 4).

Masking artefacts in the choroidal layers may be caused by blocked flow signals from overlying hyper-reflective structures, slow flow, which is below the detectable threshold or a possible segmentation error. ${ }^{13,15}$ On the other hand, unmasking artefacts are seen when areas of RPE atrophy allow the back-scatter, decorrelation signal of the underlying choroidal vessels to be seen as areas of increased flow within the choroid (Figure $3 \mathrm{c}) .{ }^{13}$ On crosssectional OCTA, the high flow signal is seen directly under the areas where the hyperreflective RPE band is disrupted (Figure 3c). On en face OCTA, the boundaries of the high flow area sharply correspond to the areas of RPE loss and this a further confirmed on comparison to fundus autofluorescence (FAF), where the high flow area corresponds to the area of hypo-autofluorescence due to RPE atrophy (Figure 3c). Other artefacts described such as the fringe washout effect and the stromal decorrelation signal, may help explain the differences in the vascular appearance in normal eyes of the choroidal vessels, which appear dark compared to the surrounding stroma versus retinal vessels that appear bright. ${ }^{13}$

\section{OCTA in age-related macular degeneration and other choroidal diseases}

Many studies have evaluated OCTA in the diagnosis and monitoring of treatment response in neovascular AMD.

\section{OCTA findings in choroidal neovascularisation (NV)}

\section{Type 1 NV}

OCTA may allow better visualisation of the vascular structure of the type $1 \mathrm{NV}$ compared to FA, as there is less masking from the overlying RPE and the vasculature is
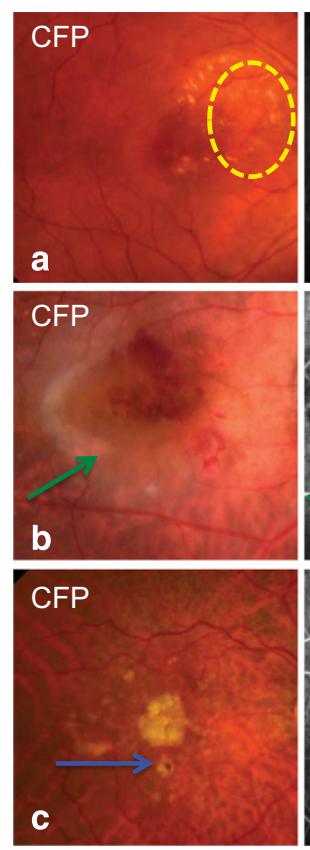
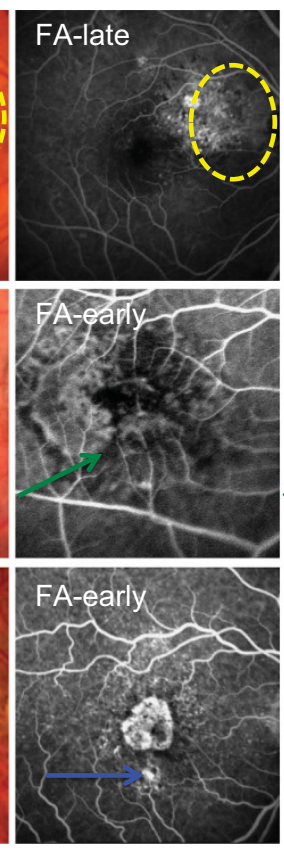
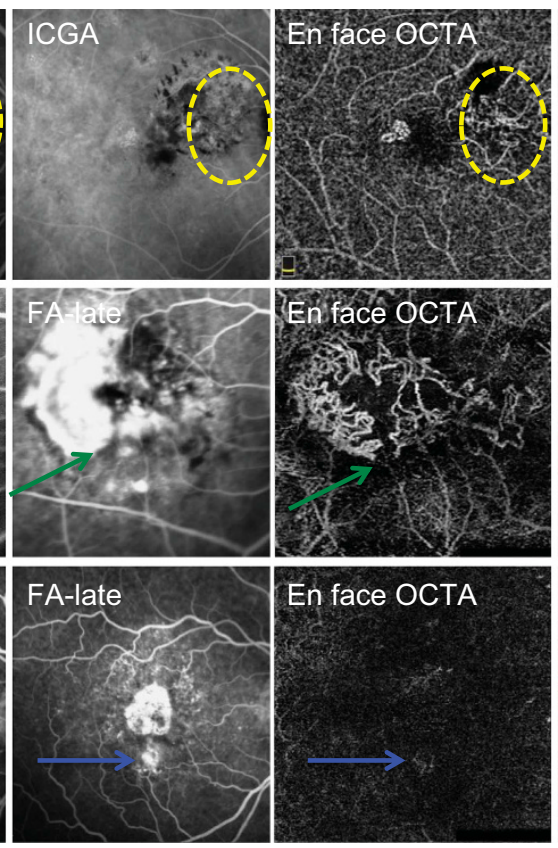

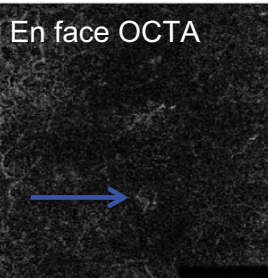

En face OCTA
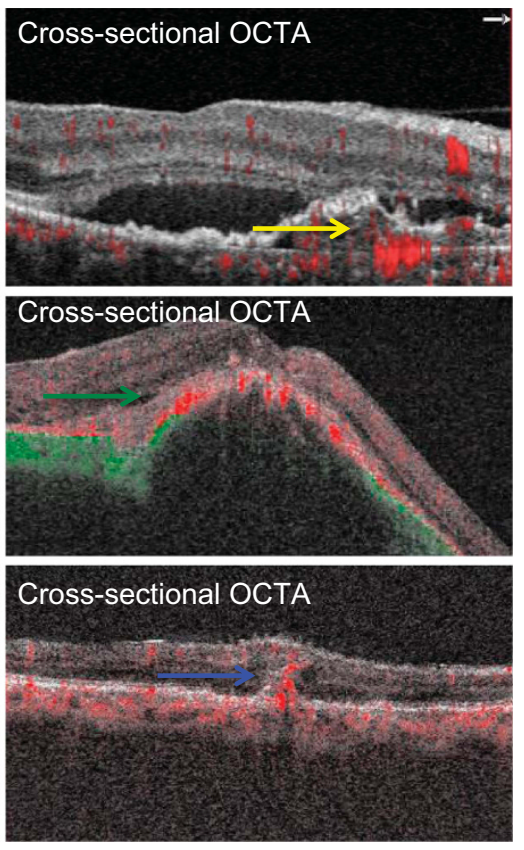

Figure 4 Multimodal images including OCTA images of the 3 subtypes of neovascular age-related macular degeneration. (CFP, colour fundus photo; FA, fundus fluorescein angiography; ICGA, indocyanine green angiography; OCTA, topical coherence tomography angiography; OCT, optical coherence tomography). (a) Type 1 neovascularisation (NV) (yellow arrows) with a vascularised pigment epithelial detachment seen on CFP, stippled hyperfluorescence and late leakage on FA, a plaque on ICGA and a vascular network seen on en face OCTA with a corresponding area of abnormal flow seen under the RPE on cross-sectional OCTA. (b) Type 2 NV (green arrows) with a greyish membrane seen on CFP, early lacy hyperfluorescence with late leakage seen on FA and a vascular network seen on en face OCTA with abnormal flow seen above the RPE on cross-sectional OCTA. (c) Type 3 NV (blue arrows) seen with associated atrophy on CFP, pinpoint leakage on FA with an area of abnormal flow on en face OCTA corresponding to a linear area of abnormal flow in the deep retina seen on cross-sectional OCTA seen below a large patch of geographic atrophy. 


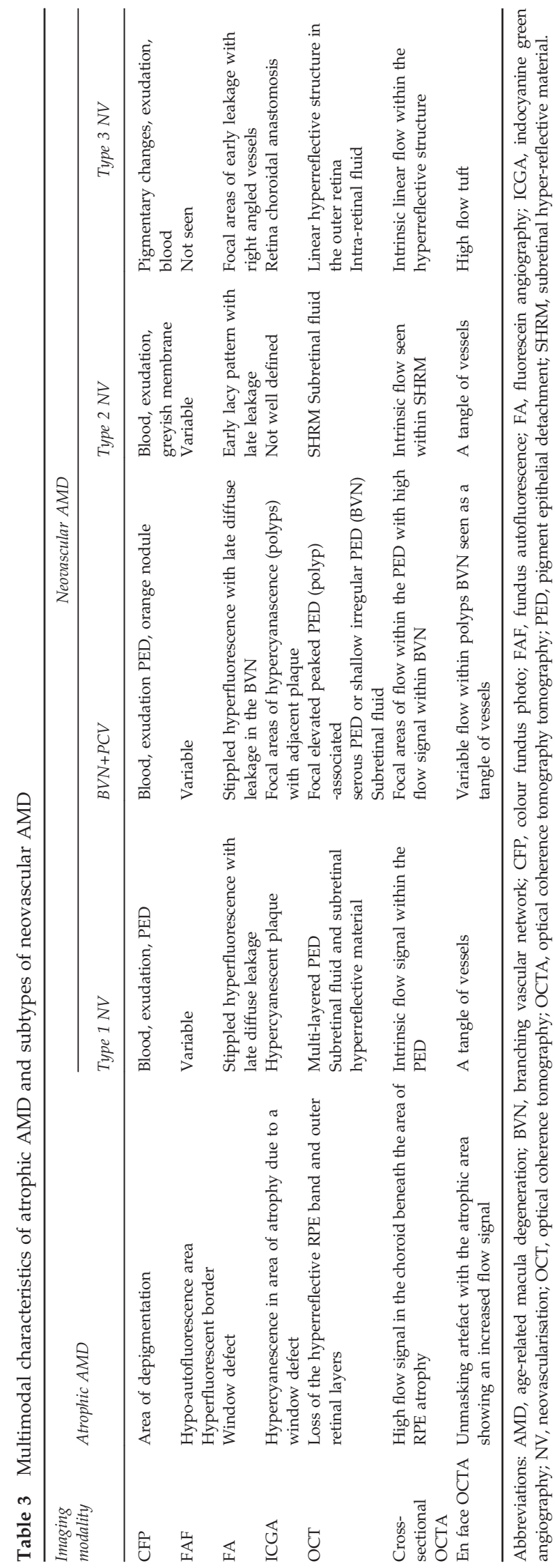

not obscured by dye leakage. ${ }^{16}$ The OCT appearance of a type $1 \mathrm{NV}$ is characterised by a vascularised pigment epithelial detachment (PED) with an irregular surface and hyperreflective contents. On cross-sectional OCTA, intrinsic flow is seen within the contents of the PED in the sub-RPE space (Figure 4a). ${ }^{17}$ In the corresponding en face OCTA, typically, type $1 \mathrm{NV}$ appears as a well-defined tangle of vessels (Figure 4a). ${ }^{17-19}$ Compared to FA, previous retrospective case series have reported that OCTA can detect type $1 \mathrm{NV}$ in $67-100 \%$ of cases. ${ }^{18-20}$ Compared to mid- or late-phase ICGA, the appearance of Type $1 \mathrm{NV}$ on OCTA has been noted to occupy significantly smaller areas (Table 3). ${ }^{21}$

\section{Type $2 \mathrm{NV}$}

An active type $2 \mathrm{NV}$ appears as subretinal hyperreflective material above the RPE with intrinsic flow signals on cross-sectional OCTA (Figure 4). ${ }^{15,22}$ The hyperflow patterns detected, that were described as either a glomerulus or a medusa shape, were associated with a thicker main vessel branch connected to the deeper choroid. ${ }^{22} \mathrm{~A}$ dark halo surrounding the lesion was thought to correspond to masking from surrounding, blood, exudation or subretinal fibrosis. ${ }^{22}$ Of note, the high flow signal was also shown in some cases to cause a projection artefact onto the deeper choriocapillaris layer. ${ }^{22}$

Mixed type 1 and type 2 lesion can be observed as abnormal flow seen both above and below the RPE on cross-sectional OCTA (Figure 5b). ${ }^{23}$ By varying the depth of segmentation, both the more superficial subretinal type 2 component and the deeper sub-RPE type 1 component can be seen on en face OCTA as a vascular network. A previous paper described a larger decrease in the area of the type 2 component compared to the type 1 component in response to anti-VEGF therapy. ${ }^{23}$

\section{Retinal angiomatous proliferation (Type $3 \mathrm{NV}$ )}

Typical OCT findings of type $3 \mathrm{NV}$ show a linear hyperreflective structure extending from the outer retina to the inner retinal layers, with or without PED. Cross-sectional OCTA of type $3 \mathrm{NV}$ showed intrinsic flow within this structure and two patterns of flow were observed; either a discrete intra-retinal flow signal or a linear flow signal that extended from the intra-retinal areas deep through to the RPE band (Figure 4c). ${ }^{24}$ En face OCTA of the type 3 $\mathrm{NV}$, showed a bright high flow tuft of microvessels originating from the deep capillary plexus in the outer retina. ${ }^{24,25}$ Distinct neovascular complexes could only be imaged in $34 \%$ of eyes, all of which showed signs of activity on OCT..$^{25,26}$ 


\section{Progression of neovascularisation in response to treatment}

Various terminologies have been proposed to describe features that reflect different stages or level of activity within a neovascular network. However, there is lack of standardisation and validation, so these terms are likely to undergo further refinement. While OCTA cannot evaluate the presence of leakage or exudation, changes in pattern of vasculature on OCTA have been reported as the NV evolves from active to inactive stages.

Characteristic features suggestive of an active NV include presence of a tangle of vessels in a well-defined shape (lacy-wheel or sea fan), branching, numerous tiny capillaries, the presence of anastomoses or loops, the presence of a peripheral arcade and the presence of a hypointense halo (Figure 6). ${ }^{27}$ In contrast, inactive chronic NVs have larger more mature vessels, a 'dead tree' appearance with the absence of the anastomoses, loops and peripheral arcades. ${ }^{27}$ After intravitreal anti-VEGF therapy NV showed a decrease in vessel density, vessel fragmentation and the loss of peripheral capillaries after 1 week with recurrence of the peripheral anastomosis and increased capillary density at 4 weeks. ${ }^{28,29}$ Finally, chronic $\mathrm{NV}$ may show little anatomical response to anti-VEGF (Figure 6b). ${ }^{18}$ On OCTA, the lesion area and vessel density have been observed to remain unchanged and the vascular tangle may develop a pruned tree appearance. ${ }^{18,25}$ These fibrovascular PEDs that had undergone multiple previous treatments, demonstrated
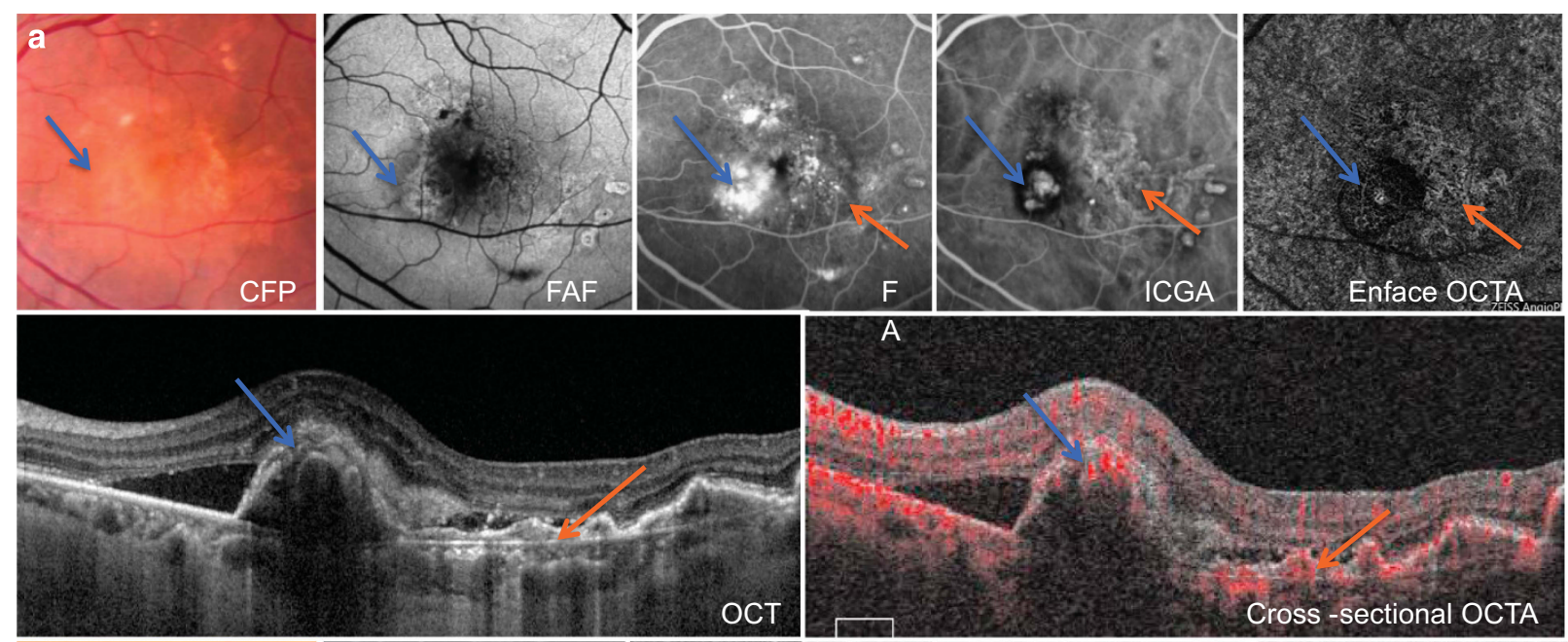

A
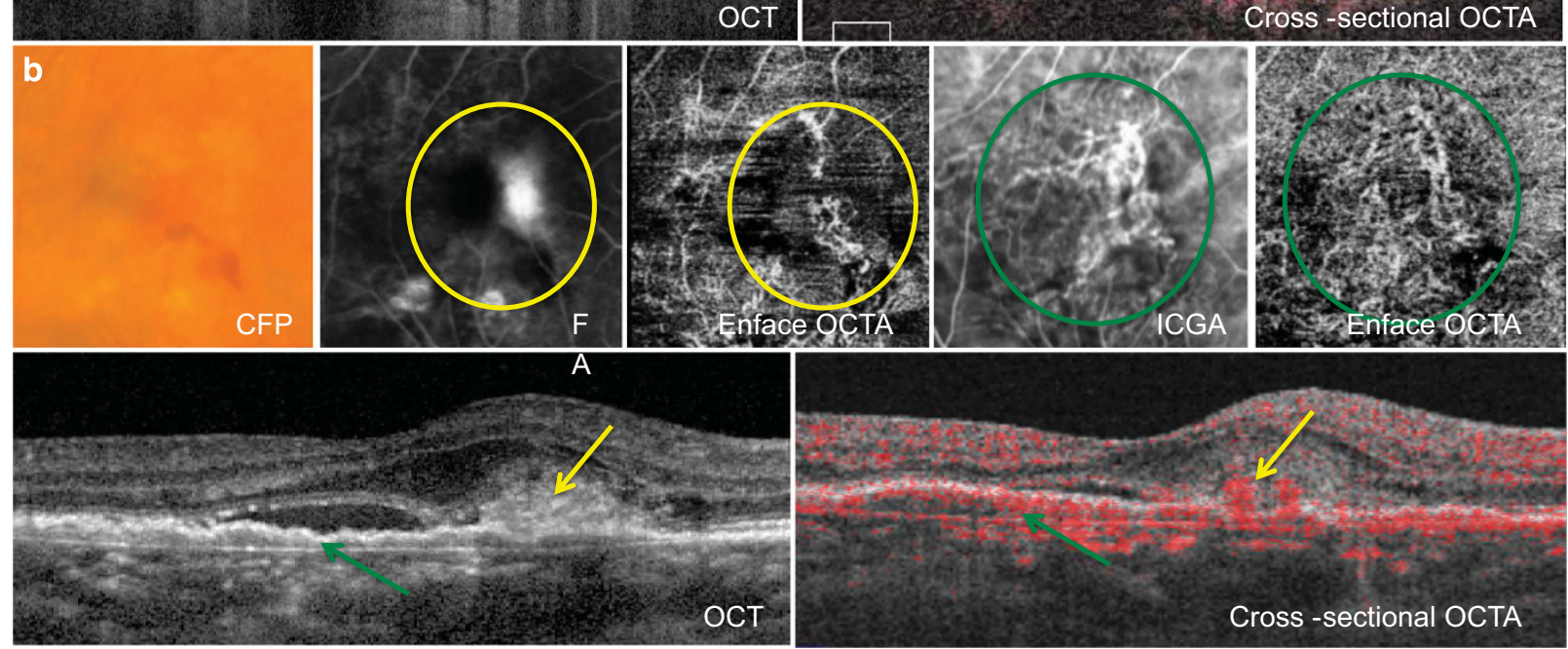

Figure 5 OCTA images showing different neovascularisation (NV) responses to treatment with intravitreal anti- vascular endothelial growth factor therapy (IVT). (a) En face OCTA (top row) shows after one IVT, there is reduction in the overall size of the type 2 NV with the regression of the smaller peripheral anastomosis leaving the larger calibre vessel trunks. After three IVTs the lesion size remains stable with the persistence of the larger calibre vessel trunks with a reduction in the dark halo surrounding the vascular lesion. Corresponding cross-sectional OCTA (second row) that show the reduction in the area of abnormal flow (red overlay) during the course of treatment. (b) En face OCTA with colour-coded density mapping showing the reduction in size of the type 1 NV (red) from baseline and after six IVTs with corresponding cross-sectional OCTAs showing a reduction in abnormal flow (red overlay) from baseline. 
prominent vascular loops and anastomotic connections and showed trunk feeder vessels of a large diameter with limited branching patterns. ${ }^{30}$

In response to anti-VEGF therapy, type $3 \mathrm{NVs}$ on OCTA showed a significant regression in the small calibre tufts in all eyes, with a reduction in median lesion area and exudation. ${ }^{6,26}$ In $29 \%$ of eyes, the high flow lesion became undetectable after a single intravitreal anti-VEGF injection, however in $65 \%$ of eyes there was persistence of the large feeder vessels. ${ }^{6}$ Longitudinal imaging of type 3 $\mathrm{NV}$ also showed that OCTA could detect changes in the vascular complex even before the presence of exudation seen on $\mathrm{OCT}$, and this may represent early recurrence. It was also noted that OCTA enabled the distinction between hyper-reflective vascular structures of the type 3 NV from other surrounding hyper-reflective foci devoid of flow, which may correlate to pigment migration. ${ }^{5}$

\section{OCTA findings in polypoidal choroidal vasculopathy (PCV) and other pachychoroid conditions}

ICGA is a useful modality for diagnosing PCV. Previous studies show that OCTA is comparable to ICGA for the detection of BVN. ${ }^{31-36}$ In contrast, the rate of polyp detection by OCTA was much more variable ranging from 17 to $85 \%$. $^{33,34,36,37}$ Using cross-sectional OCTA, most studies report the BVN to be in the sub-RPE space between the RPE and Bruch's membrane; ${ }^{31-33,35}$ however, in one study, some BVNs associated with PCVs were located deeper within the choroid (Figure 5a). ${ }^{33}$ En face OCTA of the BVN often show networks of vessels in much more detail than ICGA (Figure 5a). ${ }^{31-36}$ Crosssectional OCTA of the polyp, showed patchy flow signals within the polyp with the lumen being largely devoid of flow signals. ${ }^{31,33,35}$ En face OCTA imaging of the polyps was reported to show a more common hypoflow round structure $(75 \%)$ or less common $(25 \%)$ hyperflow round structure surrounded by a hypointense halo. ${ }^{36}$ Polyp area measured on OCTA was also noted to be consistently smaller when compared to ICGA. ${ }^{37}$ Some authors hypothesize that the slow or turbulent flow within the polyp may explain the hypoflow appearance.

One study using ss-OCTA imaging showed that in response to anti-VEGF therapy and in some cases combined photodynamic therapy, there was a reduction in flow within the PCV complex in most eyes. ${ }^{34}$ In several eyes, despite the improvement in exudation, the ss-OCTA appearance of the vascular network was unchanged. ${ }^{34}$ Changes in the appearance of the vascular network, which may represent early recurrence on OCTA may occur even without significant changes on OCT. ${ }^{34}$ Despite quiescence of lesions, as determined on OCT by the absence of exudation, $88 \%$ showed the persistence of flow signals within the vascular network and this may be a risk factor for recurrence. ${ }^{34}$

For all NVs and PCV, longitudinal changes in the flow detected on OCTA appeared to highly correlate with level of exudation assessed by structural OCT. In addition, many studies have now demonstrated that the flow signal often persists even though there is absence of fluid, or within a fibrosed scar. ${ }^{38}$

\section{Additional OCTA findings in AMD}

Studies based on OCTA have reported that $6-15 \%$ of eyes with chronic CSC have an associated type $1 \mathrm{NV}$ often seen as a shallow irregular PED. ${ }^{20,39}$ One study reported that OCTA was more sensitive at detecting vascularised PEDs associated with chronic CSC when compared to dye angiography. ${ }^{40}$

Quiescent NV refers to NV detected on conventional angiography such as FA or ICGA, which shows the absence of exudation. ${ }^{41}$ FA shows an ill-defined hyperfluorescent lesion with no leakage, while ICGA shows the presence of a hypercyanescent 'plaque'. ${ }^{41}$ OCTA was reported to have a sensitivity of $81.8 \%$ in quiescent NV detection. ${ }^{41}$ Another study examining eyes with intermediate AMD with the fellow eye having neovascular AMD, showed $27 \%$ of eyes with intermediate AMD had the presence of a 'plaque' on ICGA and a corresponding network of vessels on ss-OCTA. ${ }^{42}$ Another similar study, showed $6 \%$ of eyes were found to have the presence of type $1 \mathrm{NV}$ on OCTA, despite no leakage on FA and exudation on OCT. ${ }^{43}$ The clinical significance of these non-exudative, vascular networks remains to be determined and it has been suggested that they develop in response to retinal ischaemia. It has also been noted that some of these non-exudative networks will develop frank exudation during follow-up and thus represent the first signs of early CNV development. OCTA now provides a method to repeatedly image non-invasively these networks of dormant vessels and thus provide insights into the natural history of such lesions.

In eyes with the presence of subretinal fibrosis secondary to neovascular AMD, $94 \%$ of eyes showed the presence of abnormal flow signals within the area of fibrosis. ${ }^{38}$ Further longitudinal studies are required to determine the significance of these findings.

In early AMD, previous studies suggest there may be a generalised reduction in choriocapillaris density compared to normal age-matched controls. ${ }^{44}$ Due to the shadowing effect of drusen and PEDs, ss-OCTA has been suggested as the modality of choice due to better penetration and less shadowing. ${ }^{42-44}$ In advanced AMD with geographic atrophy (GA), due to a loss in the RPE and choriocapillaris, the changes in the underlying choroid are well seen on OCTA. ${ }^{44}$ Another study using 

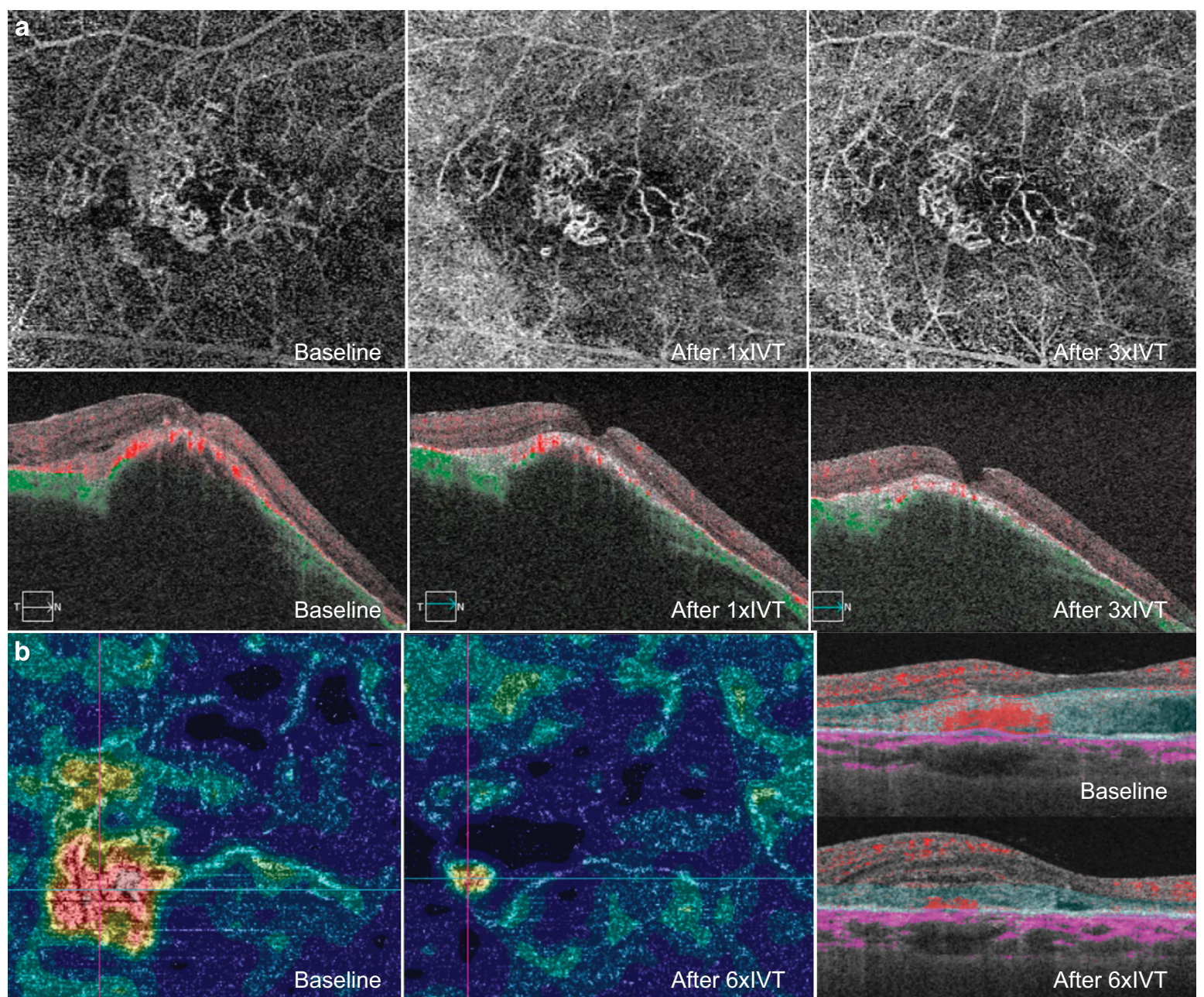

Figure 6 Multimodal images including OCTA images of mixed subtypes of neovascular age-related macular degeneration. (CFP, colour fundus photo; FAF, fundus autofluorescence; FA, fundus fluorescein angiography, ICGA =indocyanine green angiography; OCTA, topical coherence tomography angiography, OCT = optical coherence tomography). (a) Type 1 neovascularisation (NV) with polypoidal choroidal vasculopathy. Polyps (blue arrows) seen as orange nodules on CFP, focal leakage on FA, clusters of hypercyanescence on ICGA and a focal area of increased flow surrounded by a halo of decreased flow signal on en face OCTA with a corresponding area of abnormal flow directly under the RPE seen on cross-sectional OCTA. An associated branching vascular network or type $1 \mathrm{NV}$ (orange arrows) seen as stippled hyperfluorescence on FA, a plaque on ICGA and a vascular network on OCTA corresponding to shallow, irregular pigment epithelial detachment containing abnormal flow seen on cross-sectional OCTA. (b) A mixed type 2 and type $1 \mathrm{NV}$ with the subretinal type 2 component (yellow circles and arrow) and the subretinal pigment epithelial type 1 component (green circles and arrows).

ss-OCTA, reported that both focal and diffuse choriocapillaris flow impairment occurred in eyes with both nascent GA and drusen associated GA. ${ }^{45}$ In addition to the choroidal circulation, AMD can also cause vascular density reductions in the superficial and deep retinal plexuses when compared to controls. ${ }^{46}$

\section{OCTA in eyes with high myopia}

OCTA imaging in eyes with pathological myopia may help differentiate between complications such secondary choroidal neovascularisation where an abnormal flow is seen within the subretinal hyper-reflective material on cross-sectional OCTA corresponding to a hyperflow vascular network seen on en face OCTA from a simple haemorrhage where no flow or vascular network is observed. ${ }^{47}$ Previous studies have also indicated that in highly myopic eyes where general thinning of the retinal and choroidal layers are common, OCTA shows an overall reduced retinal capillary and choriocapillary density. ${ }^{48,49}$ However, OCTA imaging in eyes with high myopia is challenging due to the steep curvature of the staphyloma causing poor focus or segmentation errors, areas of myopic degeneration can 
also lead to unmasking artefacts and difficulty imaging choroidal flow. ${ }^{50}$

\section{OCTA in retinal vascular diseases}

OCTA in the imaging of vascular diseases has a few important applications. In the macular region, OCTA allows good delineation of the foveal avascular zone (FAZ) ${ }^{51-54}$ and allows the detection of macular ischaemia and microaneurysms (Figure 7). In the peripheral retina, areas of capillary dropout and the detection of neovascularisation at the disc and elsewhere can also be imaged well on OCTA (Figure 8).

\section{Diabetic eye disease}

Microaneurysms can be identified in the superficial and deep retinal capillary plexi and appear as focally dilated saccular or fusiform capillaries (Figure 7a). Studies to-date suggest that there is often disagreement between identification of microaneurysm on OCTA and FA and even between different OCTA platforms. ${ }^{52}$ Not all microaneurysms seen on FA can be found on OCTA, and vice versa some of the dilated capillary changes on OCTA that resembled microaneurysms are not found FA. ${ }^{55}$ Most studies noted more microaneurysms on FA than OCTA, ${ }^{52,56}$ however, Peres et al noted that OCTA of the DCP had more microaeurysms then either FA or the SCP on OCTA.

Comparison of FA and OCTA has demonstrated that OCTA allows better discrimination of the FAZ and parafoveal microvasculature than FA, in particular for FAZ disruption, enlargement and capillary dropout ${ }^{52}$

(Figure 7b). In diabetic retinopathy, the outline of the FAZ may become irregular, with enlarged perivascular spaces and disruption of the capillary ring. The increase in FAZ area, present in both the superficial and deep retinal plexi, can precede the development of clinical diabetic retinopathy, suggesting that diabetic eyes show impairment of the retinal microcirculation before retinopathy develops. ${ }^{57,58}$ The FAZ area was also found to increase with the presence of clinically significant

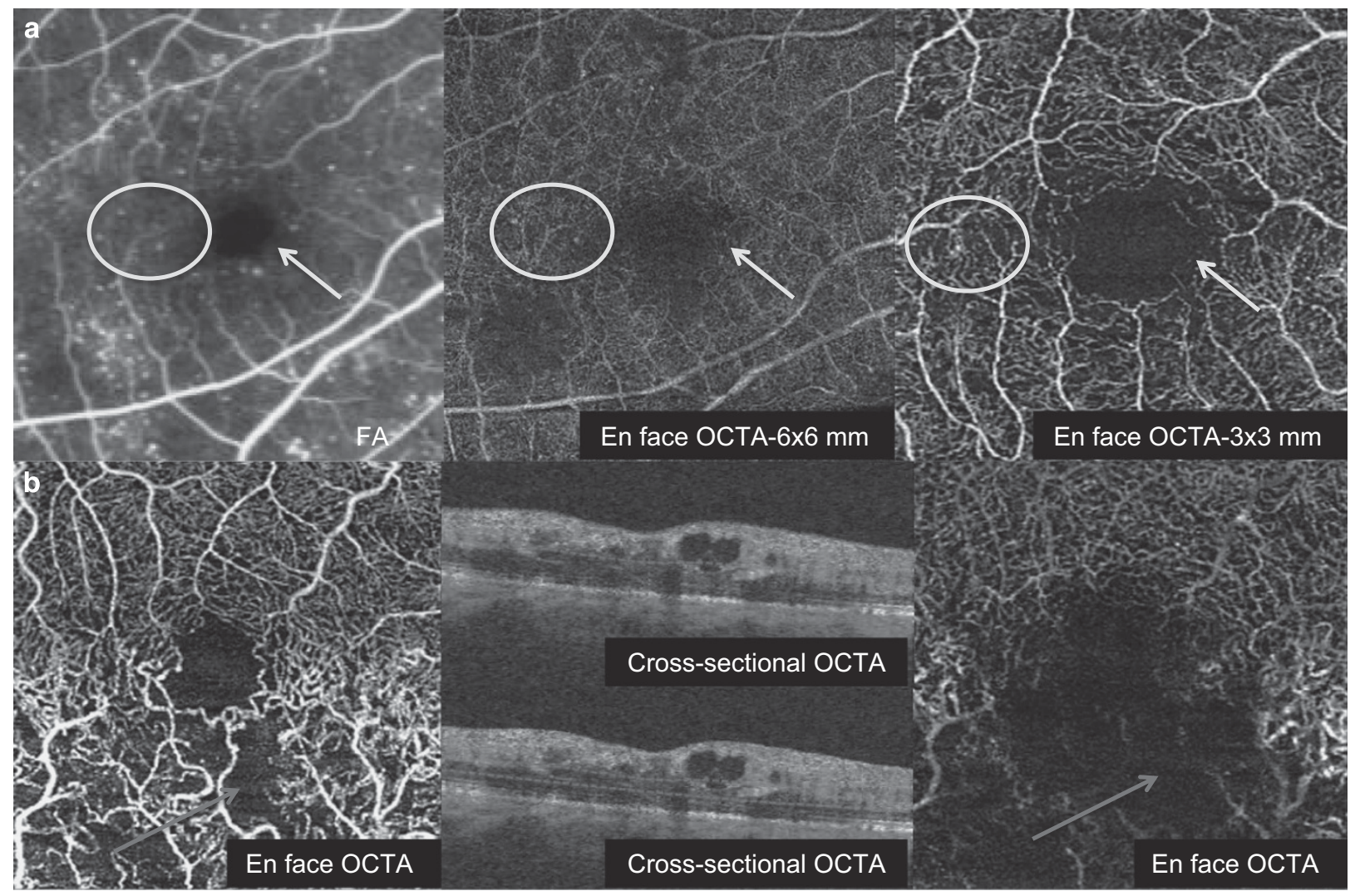

Figure 7 OCTA features in diabetic retinopathy. (a) An eye with severe non-proliferative diabetic retinopathy with microaneurysms surrounding the fovea as seen on fluorescein angiography (left) and the corresponding $6 \times 6$ (middle) and $3 \times 3$ (right) en face OCTA of the superficial segmentation. An enlarged FAZ is also noted (yellow arrows). (b) An eye with diabetic macula oedema and an enlarged FAZ (green arrow) with disruption of the normal vasculature inferiorly as seen on en face OCTA with superficial segmentation (left) and corresponding cross-sectional OCTA middle (top) and similarly with deep segmentation (right and middle bottom). Both the cystic spaces from diabetic macula oedema and areas of non-perfusion are seen as dark areas on the deep segmentation en face OCTA. A full color version of this figure is available at the Eye journal online. 


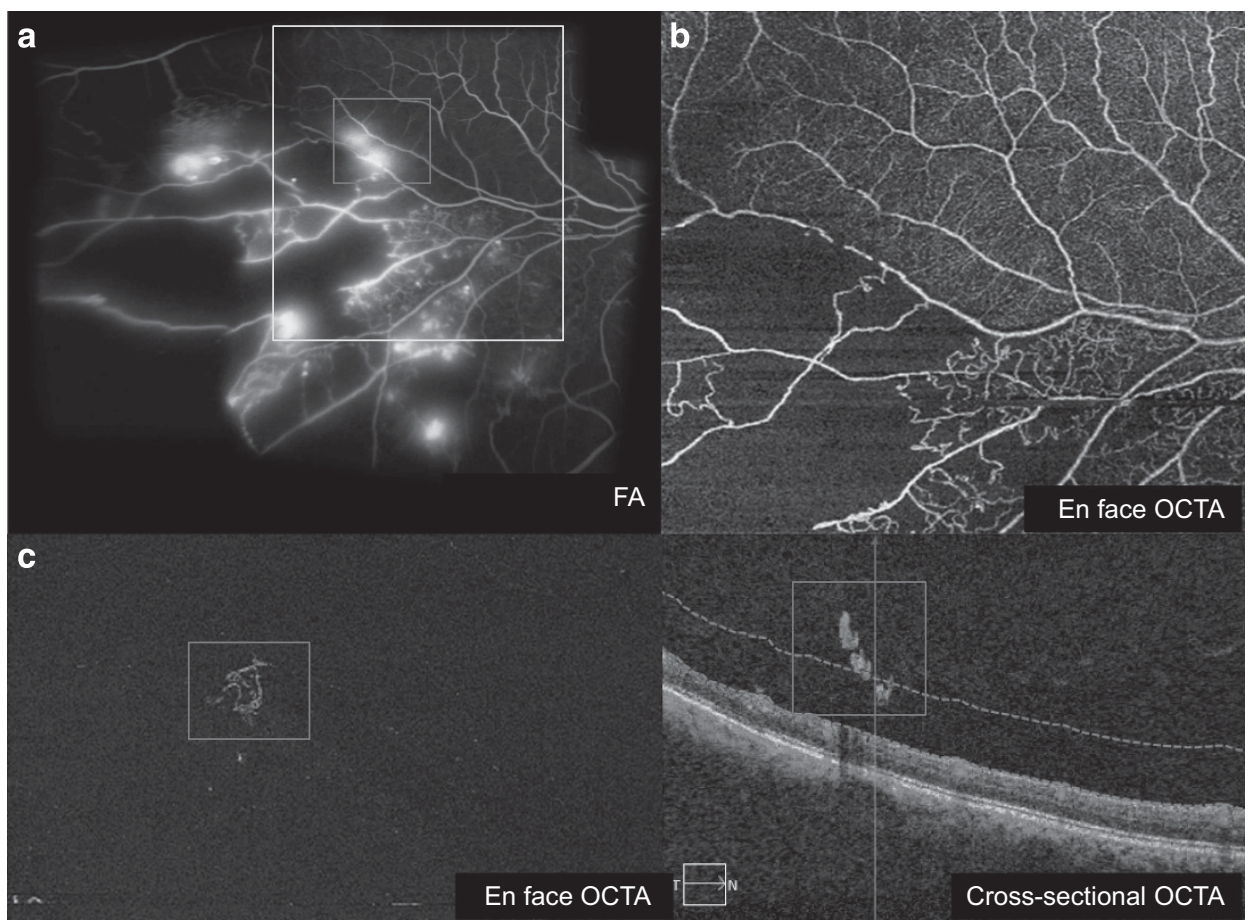

Figure 8 OCTA features of branch retinal vein occlusion. (a) Fundus fluorescein angiography (FA) showing an ischaemic branch retinal vein occlusion with neovascularisation and areas of capillary non-perfusion. (b) The areas of non-perfusion corresponding to the FA (yellow box) are seen clearly on en face OCTA. (c) An area of neovascularisation leaking on FA (green box) is seen on en face OCTA as a small vascular tuft of high flow growing into the posterior hyaloid as seen on cross-sectional OCTA. A full color version of this figure is available at the Eye journal online.

macular oedema (Figure 7b); however, there was no statistically significant difference in FAZ area between eyes with non-proliferative and proliferative diabetic retinopathy. It is important to note that variations in axial length can affect the retinal vessel magnification on OCTA. Adjustment can be made if axial length measures are available. Some strategies to quantify the irregularity of the FAZ independent of axial length include an acircularity index, defined as the ratio of the perimeter of the FAZ to the perimeter of a circle with an equal area and an axis ratio, defined as the ratio between the major and minor axis of an ellipse defined by custom software. ${ }^{53}$ Krawitz et al demonstrated that both the acircularity index and axis ratio increase with diabetic retinopathy severity and may have potential to characterise disease severity. ${ }^{53}$ These structural changes on OCTA in diabetic eyes have also been correlated with function.

Balaratnasingam et al described the correlation between larger FAZ area and worse visual acuity in eyes with diabetic retinopathy. Samara et al found that visual acuity also correlated with FAZ area, vessel area density and vessel length density on OCTA. ${ }^{59}$

OCTA can also identify neovascularisation associated with diabetic retinopathy or retinal vein occlusions (Figure 8). Serial OCTAs can been used to monitor the change in area of disc neovascularisation in response to treatment with anti-VEGF injections. ${ }^{60}$ When appropriately used, OCTA can also identify the presence of ischaemia and neovascularisation in the retina mid periphery; and differentiate new vessels which tend to be anterior to the internal limiting membrane, from collaterals and intra-retinal microvascular abnormalities. ${ }^{61}$

Quantitative tools have been developed to quantify areas of retinal perfusion on OCTA. Capillary fall out in the area surrounding the FAZ can be readily identified, and may exceed that identified on FA, as these areas can be masked by diffuse fluorescein leakage. ${ }^{62}$ Retinal vasculature on OCTA can be skeletonised or binarised to define the total capillary length or luminal area. Various measures of capillary density based on ratio of luminal area to total area have also been described. ${ }^{63-66}$ Good repeatability and reproducibility of FAZ area and capillary density on OCTA have been demonstrated in normal eyes. ${ }^{67}$ In normal eyes, with increasing age, capillary density was found to decrease while the FAZ area increases. ${ }^{67}$ Studies have consistently described lower capillary density in diabetic eyes compared with controls in both the deep and superficial layers. There was also a consistent trend of decreasing capillary density with retinopathy severity ${ }^{63,64,66}$ Automated algorithms to detect the avascular area on OCTA was not only shown to 
be highly repeatable and reproducible with the coefficient of variation reported to be $<7.0^{\circ}{ }^{63}$ but could also be used to distinguish mild NPDR from normal eyes. ${ }^{65}$ Other authors who have examined capillary density and vascular measures such as fractal dimension have also demonstrated a similar trend with severity of DR. ${ }^{64,66}$ Ting et al also demonstrated that hyperlipidemia, smoking and renal impairment were associated with capillary density decrease while increased $\mathrm{HbA} 1 \mathrm{c}$ and renal impairment were associated with a increased fractal dimension in diabetic eyes, suggesting the link between vascular risk factors and preclinical retinal microvascular changes seen on OCTA. ${ }^{66}$

In diabetic macular oedema (DMO), OCTA has been used to assess the baseline characteristics as well as response to anti-VEGF injections (Figure $7 \mathrm{~b}$ ). Lee et al found that DMO eyes had more microaneurysms in the capillary plexus, a lower vascular flow density and a larger FAZ area in the DCP then eyes without DMO. DMO eyes, which were poor responders to anti-VEGF treatment had a significantly larger FAZ area and more microaneurysms in the deep capillary plexus on OCTA compared to eyes that responded well to anti-VEGF treatment. This suggests that the deep capillary plexus is important in DMO occurrence and may be a useful prognostic tool for predicting anti-VEGF treatment response in DMO. Another study examining OCTA in DMO did not demonstrate any change in FAZ area after the treatment of macular oedema with a single injection of anti-VEGF. ${ }^{54}$

\section{Retinal vascular occlusions}

OCTA can be used to confirm the clinical diagnosis of both retinal vein and artery occlusions. ${ }^{68,69}$ Similar to its utility in DR, OCTA is able to identify capillary nonperfusion, retinal ischaemia, collateral vessels, capillary telangiectasia and microaneurysms, in addition to delineating the FAZ in macular ischaemia secondary to retinal vein occlusion (RVO) (Figures $8 \mathrm{a}$ and b). ${ }^{61,69-74}$ It has been noted that in RVO, the microvascular changes on OCTA are more prominent in the deep retinal plexus than in the superficial plexus. ${ }^{72}$ The FAZ area and vessel density on OCTA have been found to correlate with strongly with visual acuity in RVO both before and after treatment with anti-VEGF injections. ${ }^{70,75,76}$ OCTA has also been used to follow-up RVO after treatment, and demonstrated reduction of areas of non-perfusion and capillary disruption after treatment with anti-VEGF injections. ${ }^{77}$ In retinal artery occlusion (RAO), OCTA is able to delineate the extent of macular non-perfusion and follow-up changes in the vascular flow over time, and it also revealed perfusion defects in the superificial capillary plexus, which were not seen on FA. ${ }^{68}$ The development of neovascularisation in an ischaemic RVO can also be detected on OCTA (Figure 8c).

Retinal vascular disease affects both the macular and the peripheral retinal vasculature, and therefore the constraints in imaging of the peripheral retina with OCTA will limit its utility. OCTA is also unable to identify areas of focal leakage unlike FA and is dependent on a cooperative patient with reasonable fixation to produce high-quality images. Segmentation in eyes with macular oedema can also be challenging and can affect the interpretation of the retinal capillary plexus, with as many as $22 \%$ of images being unreadable. ${ }^{54,59}$

\section{Macular telangiectasia}

In macula telangiectasia type 1 (MacTel1), a predominantly unilateral disease, previous studies have reported focal microvascular dilatation and both global and focal capillary depletion when compared to the fellow eye and normal controls. ${ }^{78}$ Volume rendered OCTA images of eyes with macula telangiectasia type 2 (MacTel2) suggested that the microvascular changes may be due to vascular invasion and retinal thinning and secondary subretinal neovascularisation originate in the retinal circulation but could infiltrate both the subretinal space and the overlying thinned retina. ${ }^{79}$ The contraction of the tissue surrounding the temporal macula in the presence of stellate arranged vessels may explain the origin of the right-angle veins. ${ }^{80}$ OCTA was also able to study the progression of MacTel2 and showed that an increase in the inter-vascular spaces, capillary rarefaction and increasing abnormal areas of anastomosis was associated with a reduced capillary density in the superficial and deep layers when compared to controls. ${ }^{81,82}$

\section{OCTA in inflammatory diseases}

Imaging in inflammatory eye diseases has a number of key roles: (1) detection, that is, to identify the presence of an inflammatory process; (2) diagnosis, that is, to identify the type of inflammation; (3) monitoring, that is, to evaluate disease activity and damage. ${ }^{83}$ Although OCTA has a role to play in all three domains, it is emerging as a particularly valuable tool in the area of monitoring. In particular, it is proving valuable in two key areas where other imaging techniques sometimes fall short: (1) the need to differentiate between active inflammatory lesions, active vascular lesions and inactive fibrotic lesions highlighted by difficult treatment decisions in conditions such as Punctate Inner Choroidopathy (PIC); and (2) the need to quantify inflammatory activity, both to stratify treatment and to monitor response to treatment. 
Review of the literature in this field identifies short case series and individual case reports, many of which highlight the additional value of OCTA as part of a multimodal approach. Although no formal prospective trials to assess its diagnostic utility in uveitis are yet available, the studies discussed below show how particular clinical indications or disease groups may benefit from the additional information provided by OCTA.

\section{Clinical scenarios}

\section{Uveitic macular oedema}

As one of the leading causes of sight loss in uveitis, uveitic macular oedema (UMO) is of particular interest, although some caution is required in OCTA interpretation as extensive oedema may hamper visualisation and induce artefact. Few studies are available thus far, but it is interesting to note that in an analysis of 25 eyes with $\mathrm{UMO}$, Kim et al report significantly lower vessel density in the deep retinal plexus in eyes with UMO (vs nonUMO uveitic eyes). ${ }^{84}$ This preliminary evidence would support the hypothesis that the leakage in UMO is not entirely due to increased permeability from inflammatory mediators, but does include an ischaemic element.

\section{Retinal vasculitis}

Assessing disease activity in retinal vasculitis is challenging. It is usually based on FA with a particular regard to leakage, but also other vascular abnormalities such as progression of ischaemia. Specific limitations of FA in this context are (1) leakage of dye in FA may limit assessment of capillary 'drop-out' due to ischaemia, and (2) damaged retinal vasculature frequently remains leaky limiting reliable assessment of disease activity. OCTA has the advantages of being able to assess both structure and perfusion of the microvasculature without being obscured by leakage. It does have the disadvantage of not directly assessing leakage, which subject to the limitations above, may still be a useful indicator of active vasculitis. In an analysis of 61 uveitic eyes (including a number with retinal vasculitis) and 94 healthy eyes Kim et al report that the superficial retinal plexus showed reduced parafoveal capillary density and reduced branching complexity in uveitic versus healthy eyes; vessel calibre was also not significantly different between uveitic and healthy eyes. ${ }^{84}$

\section{Punctate inner chorioretinopathy (PIC)/multifocal choroiditis with panuveitis (MCP) spectrum}

A significant challenge in the care of patients with PIC or multifocal choroiditis with panuveitis (MCP) is to distinguish whether new lesions are inflammatory, neovascular or both (Figure 9). This has major therapeutic implications. OCT findings may be very similar in both inflammatory lesions and CNV arising from the inner choroid/RPE with sub-RPE and subretinal involvement; although CNV may have greater heterogeneity then inflammatory lesions this is not always seen, and unlike some other types of $\mathrm{CNV}$, they are not usually associated with subretinal/sub-RPE fluid. Crucially both types of lesions may leak on FA. In a prospective case series of 17 eyes (12 patients) with suspected active CNV in the context of PIC or MCP, Levison et al reported that OCTA was able to identify CNV features in 15 eyes (11 patients). ${ }^{85}$ They noted that identification was easier in the $3 \times 3 \mathrm{~mm}$ rather than the $8 \times 8 \mathrm{~mm}$ scan. OCTA failed to identify the CNV in two eyes: in one, the CNV was a peripaillary $\mathrm{CNV}$; in the other, the $\mathrm{CNV}$ was obscured by a disciform scar with oedema. OCTA also provides a noninvasive way of monitoring the response to treatment (Figure 9).

OCTA may change our understanding of the risks of $\mathrm{CNV}$ formation in posterior uveitis. In a retrospective analysis of 18 eyes with multifocal choroiditis, Zahid et al reported that the majority of eyes $(16 / 18)$ had lesions in which flow could be detected by OCTA, a much higher rate of neovascularisation than previously reported in a series using other forms of imaging. ${ }^{86}$ In a series of 40 eyes with multifocal choroiditis (26 patients), Cheng et al report that there were 23 active $\mathrm{CNV}$ cases detected on FA, of which 20 were confirmed on OCTA; the three which could not be confirmed had been excluded due to artefact. ${ }^{87}$ The authors also imaged 34 lesions (13 eyes), which were thought to be purely inflammatory, and noted that two of these showed flow on OCTA. ${ }^{87}$

Overall OCTA would suggest that the prevalence of unrecognised neovascularisation is high in these forms of uveitis. This may have implications for treatment, such a lower threshold for treatment with anti-VEGF therapy. Furthermore these new revelations may also have relevance to some of the more acute inflammatory syndromes, which are even less commonly thought to be associated with CNV. Chen et al report on four cases of atypical MEWDS who were all noted to have type 2 neovascularisation on FA and/or OCTA. ${ }^{88}$ It is likely that ready access to non-invasive angiographic assessment will reveal greater prevalence of neovascular elements in these conditions also. It is however noted that most of these cases do not progress to clinically visible CNV suggesting that a significant proportion do involute, either spontaneously or in response to immunosuppressive therapy. 

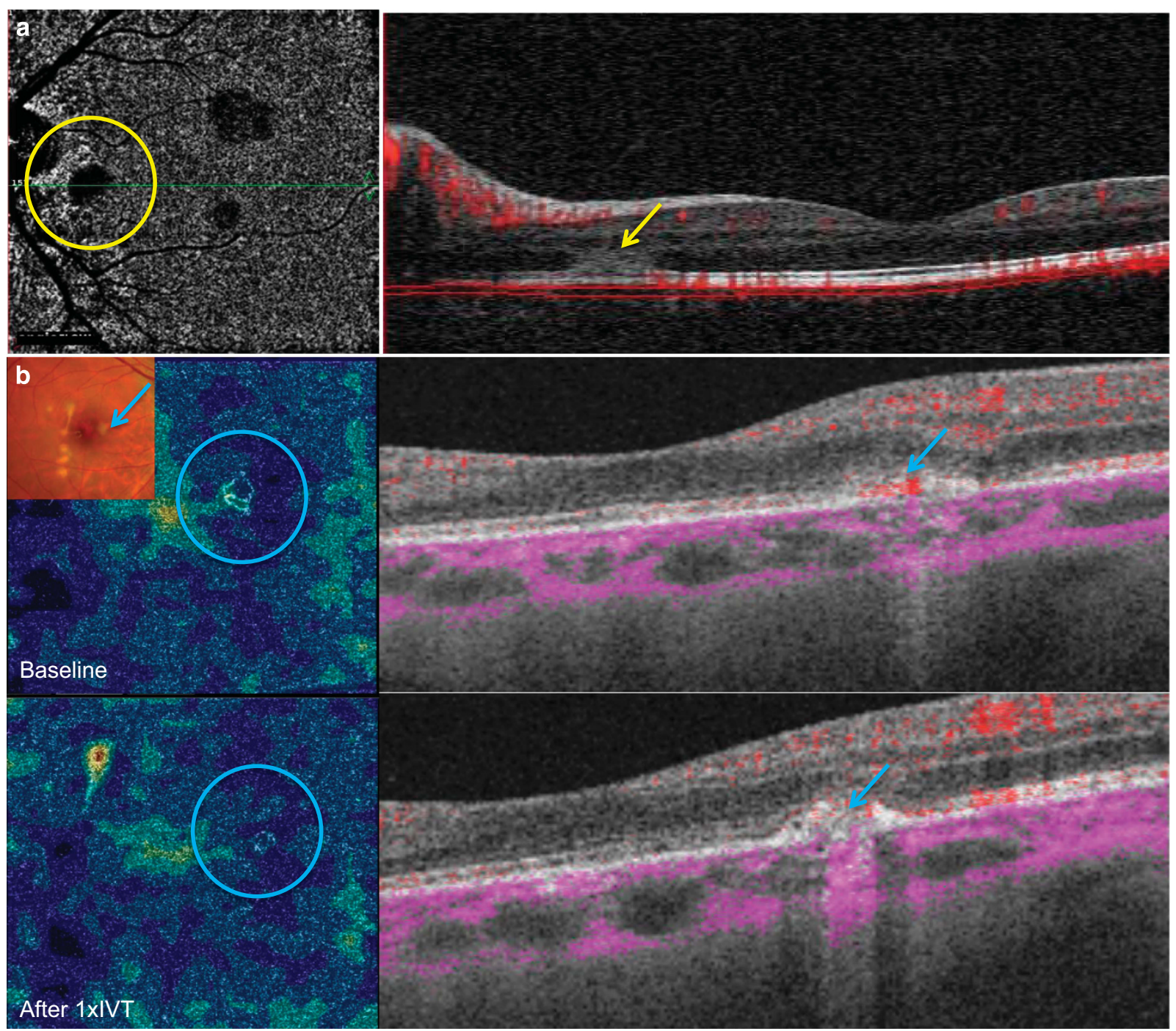

Figure 9 OCTA identifies neovascular membrane secondary to puntate inner chorioretinopathy (PIC). (a) En face OCTA shows an area of absent flow (yellow circle) on the choriocapillary segmentation seen to correspond with a hyper-reflective inflammatory lesion (yellow arrow) on cross-sectional OCTA with absent flow. (b) Another PIC lesion seen on colour fundus photo (top left), the corresponding en face OCTA shows a secondary choroidal neovascularisation (CNV) (blue circle), with the corresponding crosssectional OCTA showing an area of abnormal flow (blue arrow) on the hyper-reflective inflammatory lesion. After 1 intravitreal antivascular endothelial growth factor therapy (IVT) (bottom row), there is regression of the CNV seen on both en face and cross-sectional OCTA (blue circle and arrow).

\section{Birdshot uveitis}

In a study of OCTA findings in eight eyes (four patients) with Birdshot Chorioretinopathy (BCR), de Carlo et al reported that all eyes showed abnormalities including areas of reduced choroidal blood flow below the disrupted $\mathrm{RPE}$, retinal thinning, abnormal telangiectatic vessels and an increased capillary space; the increased capillary space was most prominent temporally. ${ }^{89}$ Additionally, capillary dilatations and loops were seen in $7 / 8$ eyes. It is worth noting that these features were present even when classic birdshot lesions were not visible on fundus photography. Roberts et al reported on 37 eyes (21 patients) with BCR vs a similar number of healthy controls, and noted that the capillary density of the full retina, and both the superficial capillary plexus and deep capillary plexus were reduced in BCR compared to the healthy controls. ${ }^{90}$ Importantly visual acuity in the BCR group was associated with a reduced capillary density, whereas FAZ area did not appear to have an effect.

\section{Behcet's Uveitis}

In a prospective comparative study of FA, SD-OCT and OCTA in Behcet's disease, Khairallah et al noted the 
additional value of OCTA in detecting vascular abnormalities in 25 patients (44 eyes) with Behcet's disease. ${ }^{91}$ They reported that perifoveal microvascular changes were noted more commonly on OCTA versus FA (96 vs 59\%), with specific abnormalities including disrupted perifoveal capillary arcades, retinal capillary under-perfusion, and rarefied, dilated, or shunted capillaries. These abnormalities were more commonly seen in the deep than in the superficial capillary plexus. Key differences in the Behcet's group versus healthy controls, included increased FAZ area of the superficial and deep capillary plexi, with lower overall capillary vessel density in the deep capillary plexus.

\section{Vogt-Koyanagi-Harada disease}

Clinically the early chorioretinal findings of acute VKH and multifocal central serous chorioretinopathy (CSC) may be similar. In a study of 24 patients $(10 \mathrm{VKH}, 14$ CSC), Aggarwal et al evaluated the ability of OCTA to identify differences that might help distinguish these entities. ${ }^{92}$ They noted that in VKH there appeared to be a true choriocapillaris flow void related to ischaemia; an important caution however is that they also noted some similar changes in the CSC group, which they ascribed to overlying SRF and PED. The elicitation of true versus artefactual flow voids will be critical to its diagnostic utility in this context.

\section{Acute posterior multifocal placoid pigment epitheliopathy (APMPPE)}

The pathogenesis of APMPPE has been a controversial area ever since its original description by Gass and his proposal that it primarily targeted the RPE. Subsequent dye-based angiography has suggested that it may be primarily a choroidal vasculitis focused on the choriocapillaris. The application of OCTA may help to elucidate this.

In their report Kinouchi et al described a single case of APMPPE, in which they noted that in the acute phase OCTA demonstrated dark areas with a lack of flow signals at the level of the choriocapillaris corresponding to the placoid lesions. They suggested that this was not likely to be simply due to 'blockage' by RPE oedema, as flow signals from the deeper choroid in these regions were still present. Similarly Salvatore et al described a single case of APMPPE, in which OCTA suggested altered flow and non-perfusion in defined islands of choriocapillaris. In both cases progressive reperfusion and visual recovery occurred over time.

Interestingly in their retrospective case series of five patients with APMPPE, Heiferman et al noted that choriocapillaris flow abnormalities extended beyond the visible lesions but cautioned against over-interpretation of the apparent flow voids immediately underlying the acute lesions due to the potential 'blockage' effect. ${ }^{93}$ They did note however that after the acute phase when visualisation of the choroid improves, residual vascular abnormalities could be seen. Their finding that these vascular abnormalities may occur outside of the areas of clinically defined involvement, may suggest that the disease process in APMPPE is more extensive than currently appreciated. ${ }^{93}$

\section{Other retinal inflammatory lesions}

OCTA may be used to identify the presence of neovascularisation in other inflammatory foci, such as isolated retinal or choroidal lesions commonly termed 'granulomata'. Pichi et al describe a case of active Bartonella, in which OCTA of a retinal granuloma illustrated a network of vessels with microvascular proliferation within the inflammatory lesion. ${ }^{94}$ Follow-up OCTA was also able to show reduction of the vascular network as the lesion responded to treatment. ${ }^{94}$

\section{OCTA in optic nerve disease}

\section{Glaucoma}

Primary open angle glaucoma (POAG) is a multifactorial optic neuropathy, possibly involving vascular dysfunction, leading to death of retinal ganglion cells and of their axons. Exploration of ocular vasculature in glaucoma has been challenging, due to various limitations in the imaging modalities; therefore, the novel developments of OCTA have raised a large interest in exploring the optic nerve microvasculature in POAG. Initial, cross-sectional studies have suggested that OCTA can be useful in evaluating the optic disc and the peripapillary retinal perfusion in glaucoma. ${ }^{95,96}$ Furthermore, these studies have shown the ability of OCTA to display attenuation of the optic nerve microvasculature in POAG, both at the optic nerve head level and at the peri-papillary area, compared to normal eyes. Thus, OCTA allows noninvasive three-dimensional visualisation of the optic nerve head vasculature, from the disc surface to the lamina cribrosa, as well as quantification of optic disc perfusion.

Conventional OCT has successfully provided objective and quantitative structural measurements in POAG, including evaluation of ganglion cell complex in the macular region and retinal nerve fibre layer (RNFL) thickness at peri-papillary regions. However, these conventional structural OCT measurements do not seem to entirely reflect the functional outcome in glaucoma. Indeed, structural loss in glaucoma has only moderate 
correlation with loss of the visual field (VF) in POAG, especially at early stages. On the contrary OCTA not only reveals reduced retinal vessel density in POAG, but OCTA findings also correlate well with the disease severity and the associated visual field loss. ${ }^{97}$ These significant vascular-functional correlations in POAG, revealed by OCTA, might be explained by a pre-apoptotic status of the retinal ganglion cells at early stages. These cells and their axons may be affected only functionally at the early stages, due to the reduced vascular supply (as shown by OCTA). Their subsequent damage may translate only later into reduced visual field sensitivity and OCTA may allow the early detection of microvascular abnormalities in the course of POAG. Indeed, OCTA can disclose decreased peri-papillary, optic nerve head and macular vessel densities, not only in early glaucoma with limited VF loss but also in pre-perimetric POAG, when standard automated perimetry is still intact, despite RNFL thinning. ${ }^{98}$ OCTA may also be helpful for exploring vascular changes in secondary optic glaucomatous optic neuropathies due to neuroophthalmic conditions, such as increased episcleral vessels pressure occurring in carotid-cavernous fistulas. ${ }^{99}$

Taken together, these initial cross-sectional, observational studies suggest that OCTA may represent a potential tool for detecting vascular abnormalities in POAG, which may translate in the future into early diagnosis and improved disease monitoring. However, these preliminary studies have several inherent (technical, methodological) limitations and most importantly, do not explain yet if the microvascular attenuation in POAG is a cause or rather a consequence of the glaucomatous condition. Longitudinal studies may clarify in the future this complex temporal relationship.

\section{Non-arteritic ischaemic optic neuropathy}

Non-arteritic anterior ischaemic optic neuropathy (NAION), the most common non-glaucomatous optic neuropathy in the elderly population, is characterised by acute, painless, typically unilateral visual loss and altitudinal visual field defect, associated with optic disc swelling. NAION is possibly caused by transient hypoperfusion in the capillary bed of the optic nerve head, which is closely connected with the choroidal vasculature-explaining the high interest of its exploration with OCTA.

OCTA may allow evaluation of the peri-papillary microvasculature in eyes with NAION, both at the acute stage (Figure 10), and after resolution of the optic disc swelling. ${ }^{100}$ In a small pilot study, exploring eyes with NAION at the acute stage (within 1 week of visual loss), OCTA imaging revealed significant segmental and global reduction of the peri-papillary vascular flow density, compared to the fellow, healthy eyes and to age-matched control eyes. In addition, OCTA may also reveal tortuous capillaries within or surrounding the optic disc in NAION, a finding clinically described as pseudoangiomatous hyperplasia. ${ }^{101}$ It is not clear yet if OCTA may be useful for the longitudinal follow-up in NAION, but preliminary data suggests that OCTA may reveal spontaneous, partial recovery of peri-papillary vascular flow densities during the natural course of the disease, in line with the limited improvement of the visual function. ${ }^{100}$ In addition, OCTA may also have the ability to evaluate progression of the disease, that is, from an infra-clinical, incipient stage of NAION to its full-blown clinical picture. ${ }^{100}$ OCTA may therefore have a potential role in monitoring the evolution of NAION.

However, OCTA has specific limitations in the evaluation of NAION. The reduction of the flow density at different layers in NAION may not reflect a primary ischaemic process, but rather ma be the result of compressive oedema, or of imaging artefacts (signal attenuation by blood and/or oedema).

\section{Optic neuritis, multiple sclerosis and optic atrophy}

OCTA has been used for the evaluation the optic nerve head microvasculature in other neuro-ophthalmic conditions causing either true or pseudo-optic disc oedema (idiopathic intracranial hypertension, Leber's hereditary optic neuropathy) or, at later stages, optic disc atrophy (after optic neuritis, NAION, or in autosomal dominant optic atrophy). ${ }^{101}$ Optic disc oedema, irrespective of its origin, may be associated with vessel tortuosity and dilated prelaminar capillary network on OCTA, but the magnitude of the associated vascular dropout may depend on the nature of the optic nerve condition and its severity. Optic disc oedema related to idiopathic intracranial hypertension may be associated with relatively preserved peri-papillary microvasculature in its early stages (Figure 2). Further longitudinal studies are needed to assess the natural history of OCTA findings in optic disc oedema.

Patients with multiple sclerosis (MS) who had previous episodes of optic neuritis display a reduced peri-papillary and parafoveal vascular flow index, compared to healthy controls, as well as compared to patients with MS without previous optic neuritis attacks. ${ }^{102,103}$ Interestingly, even in absence of optic neuritis attacks, patients with defined MS display a reduced vascular flow with OCTA, compared to controls. ${ }^{102,103}$ In patients with optic neuritis, OCTA may display residual microvascular abnormalities of the optic nerve and the macula, despite recovery of visual function after treatment. ${ }^{103}$ 

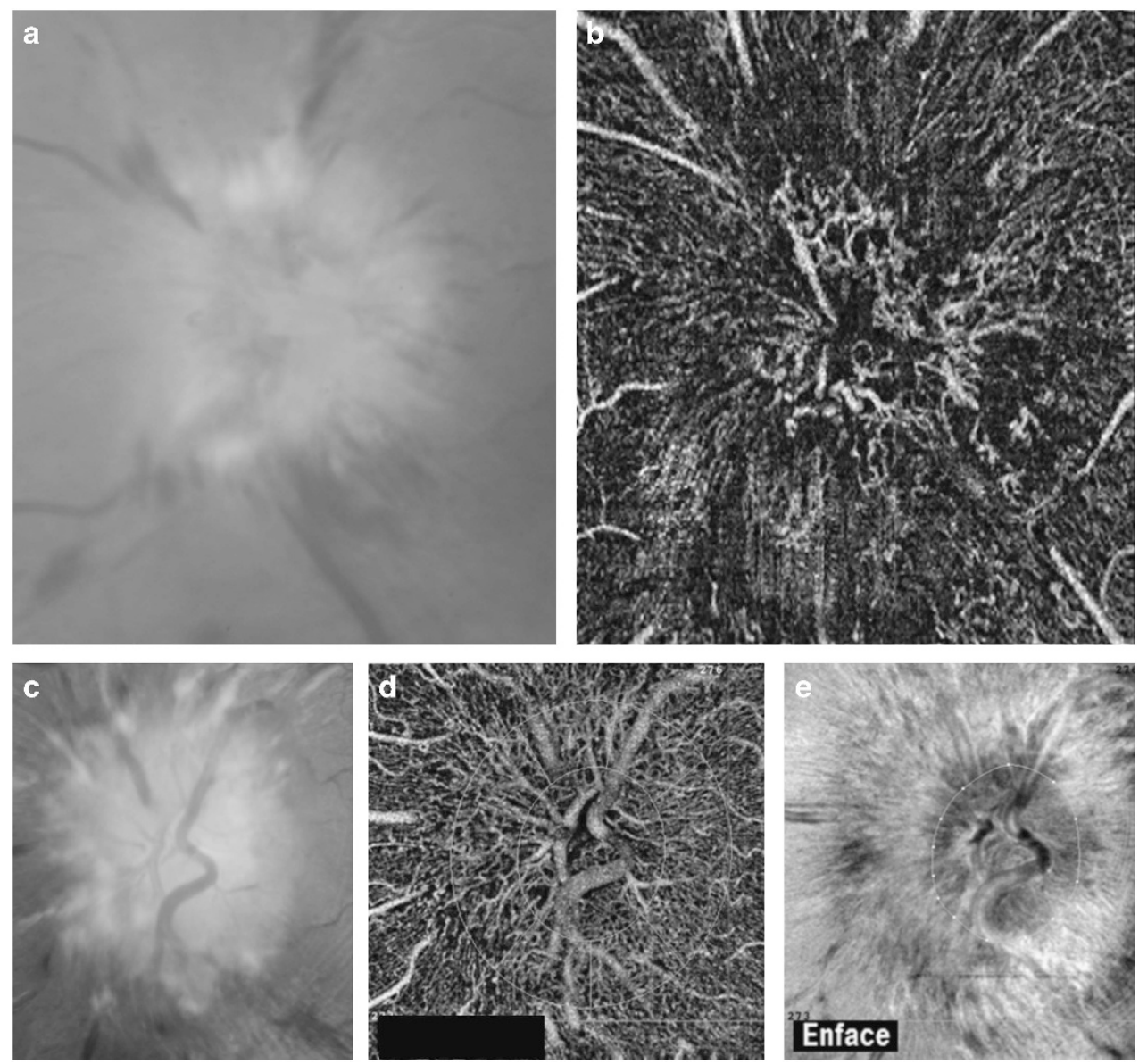

Figure 10 OCTA findings in a patient with non-arteritic ischaemic optic neuropathy and ipsilateral visual loss. The sectorial optic disc swelling (a), is associated on OCTA (AngioVue, Optovue) with tortuous radial peri-papillary capillaries and vascular dropout in the optic nerve head (b). Optic nerve head swelling in a patient with idiopathic intracranial hypertension and preserved visual function (c). Despite the severe optic disc swelling and peri-papillary haemorrhages (c), OCTA evaluation (AngioVue, Optovue), discloses only limited vascular dropout in the optic nerve head region ( $\mathrm{d}$ and e). A full color version of this figure is available at the Eye journal online.

\section{OCTA in anterior segment disease}

Angiography for the anterior segment has a variety of clinical applications, ranging from the evaluation of scleral inflammatory disorders, to the assessment of corneal vascularisation. Currently, the assessment of the anterior segment vasculature is constrained to invasive angiography techniques using FA or ICGA. However, invasive angiography techniques expose patients to potential adverse reactions. Thus, imaging and evaluation of corneal vascularisation has been limited, despite its prevalence and potential sight-threatening effects. Also, significant time and preparation is required before each ICGA or FA-imaging session, while some patients may not be suitable for this procedure at all, due to various contraindications. Thus there is an increasing role for OCTA for the anterior segment. ${ }^{104}$

The main advantage of OCTA for the anterior segment is that images are rapidly acquired using a non-contact technique. ${ }^{105}$ While the split-spectrum amplitude- decorrelation angiography system has been most commonly described for the anterior segment, other spectral domain and swept source OCTA systems have also been successfully adapted for the anterior segment. ${ }^{106}$ However, it is important to note that current OCTA systems are not specifically designed for the anterior segment but may be adapted to assess the cornea or iris vasculature. ${ }^{105}$ Thus there are several limitations such as the inability to demonstrate vessel leakage, and a limited field of view compared to the FA and ICGA. ${ }^{107}$ Moreover, as the lens had to be relatively close to the surface of the cornea for the vessels to be in focus, image acquisition was relatively easier in the temporal quadrants compared to the nasal scans. Nonetheless, it has been reported that the OCTA adapted for the cornea was comparable to ICGA for measurement of the area of corneal vascularisation in one pilot clinical study. ${ }^{108}$

Similar to the OCTA for the retina and posterior segment, there are several points to note when 
interpreting OCTA scans for the anterior segment. First, image distortions may occur due to patient movement, inclinations of the scanning plane relative due to the corneal surface. Fortunately, as each non-contact scan only requires $3-4 \mathrm{~s}$ to complete, patients are usually able to tolerate multiple scans to ensure a good quality image is achieved. Second, image artefacts and loss of signal may occur in areas of dense scarring, and be compounded by the coronal reconstruction of scans. Future improvements to the software and optimisation for the anterior segment may further improve the image resolution, before which a clinician may choose to perform ICGA in eyes with concomitant dense corneal scarring. Third, the OCTA systems for the anterior segment used do not come with an in-built motion correction for ocular saccades or micro-movements. It also does not carry an eye-tracking system with registration, which is required for comparisons in follow-up scans. Nonetheless, with the help of adjunct image analysis software, it has been found to be potentially useful for serial scans and follow-up in various clinical indications.

While recognising the current limitations of OCTA systems, there are still a wide variety of potential clinical applications for delineating the vasculature of the anterior segment (Figure 11). ${ }^{99}$ These include assessment of graft vascularisation with prognostication for graft rejection, evaluation of new anti-angiogenic treatments for corneal vascularisation, studying limbal vasculature associated with limbal stem cell deficiency, or even evaluation of bleb vascularity and morphology after glaucoma surgery (Figure 11). The ability to provide high-resolution scans of the cornea with accompanying information on the depth of abnormal vasculature, is useful for planning for procedures such as lamellar keratoplasty and fine-needle diathermy; or evaluation of peripheral corneal infiltrates or melts with the adjacent inflamed sclera and limbal vessels. Moreover, while the presence of FA or ICGA leakage influences clinical management for retinal or
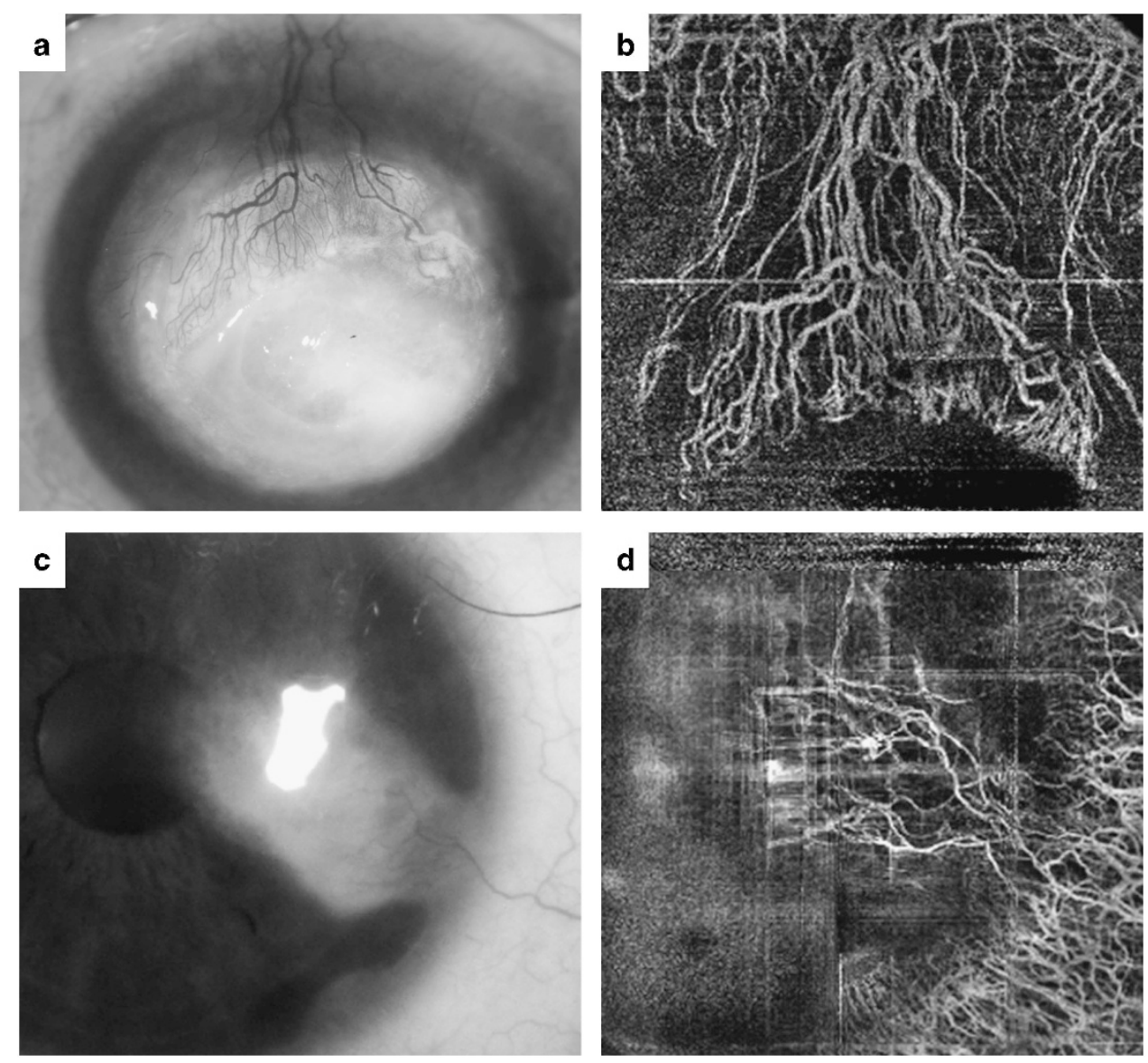

Figure 11 Optical coherence tomography angiography of the cornea. (a) Fungal keratitis with chronic inflammation and corneal vascularisation. (b) Optical coherence tomography angiography imaging may be useful to guide fine-needle diathermy and anti-VEGF therapy to reduce corneal vascularisation before corneal transplantation, and risk of corneal graft rejection. (c) Interstitial keratitis with deep corneal vascularisation. (d) Optical coherence tomography angiography reveals deeper vessels not obvious on slit-lamp microscopy. A full color version of this figure is available at the Eye journal online. 
choroidal pathology, in the anterior segment leakage blocks vessel delineation and adds limited clinical information. On the other hand, the absence of vascular flow may be a more useful clinical sign, for example in the assessment of peripheral ulcerative keratitis, a sign which is often obscured by the leakage or extravasation of dye. The progression of corneal melting and the need for systemic immunosuppression in such severe inflammatory conditions is usually preceded by vasculitis and vaso-occlusion of the limbal vessels; while recanalisation and new capillary formation may indicate response to treatment. Thus, OCTA has the potential to has an important role in detecting progression, prognostication and the management of these corneoscleral destructive diseases, which requires further studies in these specific conditions for confirmation.

\section{Other novel areas of interest}

Other upcoming applications of OCTA include the visualisation of the middle retinal plexus, the peri-papillary radial plexus as well as changes within the choriocapillaris and large choroidal vessels, previously not adequately visualised using conventional angiography; however the clinical significance of changes is unclear. The development of variable inter-scan time acquisition protocols (VISTA) may also allow variable flow rates (both slow and fast) to be detected in future OCTA platforms. ${ }^{109}$

\section{Summary}

Optical coherence tomography angiography (OCTA) has emerged as a novel, non-invasive imaging modality that allows the detailed study of flow within the vascular structures of the eye. This new technology, still in its infancy, has the potential to improve the diagnosis and monitoring of various vascular and inflammatory diseases by imaging vascular networks in greater detail than ever before. In addition, to the retina, OCTA can be used also in the anterior segment and optic nerve.

Keeping in mind the current limitations of this technology, future technical improvements and increased validation of this promising imaging modality is necessary to improve the clinical application of OCTA.

\section{Conflict of interest}

The authors declare no conflict of interest.

\section{Acknowledgements}

Dr Raymond Najjar and Dr Sourabh Sharma for help with the figures. ACT is a consultant for Zeiss and has received travel grants and sponsorship from Bayer, Allergan and Mandarin Optics. GT is a consultant for Novartis and Abbott medical, a speaker and receives travel support from Allergan, Alcon, Zeiss and Bayer; in addition to grant and travel support from Santen. AKD receive a proportion of their funding from the National Institute for Health Research (NIHR) Biomedical Research Centre based at Moorfields Eye Hospital NHS Foundation Trust and UCL Institute of Ophthalmology. PAK is funded by a Clinician Scientist award (CS-2014-14-023) from the National Institute for Health Research. The views expressed in this publication are those of the authors and not necessarily those of the NHS, the National Institute for Health Research or the Department of Health. PAK has received speaker fees from Heidelberg Engineering, Topcon, Haag-Streit, Allergan, Novartis, and Bayer. He has served on advisory boards for Novartis and Bayer, and is an external consultant for DeepMind and Optos. UC receives grant funding from Alimera Sciences, lectures and speaking events for Allergan. GC is a consultant, speaker and receives grant funding from Novartis and Bayer, is also a speaker for Allergan and Topcon.

\section{References}

1 Gao SS, Jia Y, Zhang M, Su JP, Liu G, Hwang TS et al. Optical coherence tomography angiography. Invest Ophthalmol Vis Sci 2016; 57(9): OCT27-OCT36.

2 Zhang A, Zhang Q, Chen CL, Wang RK. Methods and algorithms for optical coherence tomography-based angiography: a review and comparison. J Biomed Opt 2015; 20(10): 100901.

3 Jia Y, Bailey ST, Wilson DJ, Tan O, Klein ML, Flaxel CJ et al. Quantitative optical coherence tomography angiography of choroidal neovascularization in age-related macular degeneration. Ophthalmology 2014; 121(7): 1435-1444.

4 Kuehlewein L, Sadda SR, Sarraf D. OCT angiography and sequential quantitative analysis of type 2 neovascularization after ranibizumab therapy. Eye (Lond) 2015; 29(7): 932-935.

5 Tan AC, Dansingani KK, Yannuzzi LA, Sarraf D, Freund KB. Type 3 Neovascularization imaged with cross-sectional and en face optical coherence tomography angiography. Retina 2017; 37(2): 234-246.

6 Phasukkijwatana N, Tan AC, Chen X, Freund KB, Sarraf D. Optical coherence tomography angiography of type 3 neovascularisation in age-related macular degeneration after antiangiogenic therapy. Br J Ophthalmol 2016; 101(5): 597-602.

7 Lumbroso B, Rispoli M, Savastano MC. Longitudinal optical coherence tomography-angiography study of type 2 naive choroidal neovascularization early response after treatment. Retina 2015; 35(11): 2242-2251.

8 Ferrara D. Image artifacts in optical coherence tomography angiography. Clin Exp Ophthalmol 2016; 44(5): 367-368.

9 Spaide RF, Fujimoto JG, Waheed NK. Image artifacts in optical coherence tomography angiography. Retina 2015; 35(11): 2163-2180. 
10 Kraus MF, Liu JJ, Schottenhamml J, Chen CL, Budai A, Branchini L et al. Quantitative 3D-OCT motion correction with tilt and illumination correction, robust similarity measure and regularization. Biomed Opt Express 2014; 5(8): 2591-2613.

11 Camino A, Zhang M, Gao SS, Hwang TS, Sharma U, Wilson DJ et al. Evaluation of artifact reduction in optical coherence tomography angiography with real-time tracking and motion correction technology. Biomed Opt Express 2016; 7(10): 3905-3915.

12 Coscas G, Lupidi M, Coscas F. Image analysis of optical coherence tomography angiography. Dev Ophthalmol 2016; 56: 30-36.

13 Chen FK, Viljoen RD, Bukowska DM. Classification of image artefacts in optical coherence tomography angiography of the choroid in macular diseases. Clin Exp Ophthalmol 2016; 44(5): 388-399.

14 Huang Y, Zhang Q, Wang RK. Efficient method to suppress artifacts caused by tissue hyper-reflections in optical microangiography of retina in vivo. Biomed Opt Express 2015; 6(4): 1195-1208.

15 Dansingani KK, Tan AC, Gilani F, Phasukkijwatana N, Novais E, Querques L et al. Subretinal hyperreflective material imaged with optical coherence tomography angiography. Am J Ophthalmol 2016; 169: 235-248.

16 Malihi M, Jia Y, Gao SS, Flaxel C, Lauer AK, Hwang T et al. Optical coherence tomographic angiography of choroidal neovascularization ill-defined with fluorescein angiography. Br J Ophthalmol 2017; 101(1): 45-50.

17 Tan AC, Simhaee D, Balaratnasingam C, Dansingani KK, Yannuzzi LA. A perspective on the nature and frequency of pigment epithelial detachments. Am J Ophthalmol 2016; 172: $13-27$.

18 Kuehlewein L, Bansal M, Lenis TL, Iafe NA, Sadda SR, Bonini Filho MA et al. Optical coherence tomography angiography of type 1 neovascularization in age-related macular degeneration. Am J Ophthalmol. 2015; 160(4): 739-748. e732.

19 Inoue M, Jung JJ, Balaratnasingam C, Dansingani KK, Dhrami-Gavazi E, Suzuki M et al. A comparison between optical coherence tomography angiography and fluorescein angiography for the imaging of type 1 neovascularization. Invest Ophthalmol Vis Sci 2016; 57(9): ОCT314-OCT323.

20 Veronese C, Maiolo C, Morara M, Armstrong GW, Ciardella AP. Optical coherence tomography angiography to assess pigment epithelial detachment. Retina 2016; 36(3): 645-650.

21 Costanzo E, Miere A, Querques G, Capuano V, Jung C, Souied EH. Type 1 choroidal neovascularization lesion size: indocyanine green angiography versus optical coherence tomography angiography. Invest Ophthalmol Vis Sci 2016; 57(9): ОСТ307-OCT313.

22 El Ameen A, Cohen SY, Semoun O, Miere A, Srour M, Quaranta-El Maftouhi $\mathrm{M}$ et al. Type 2 neovascularization secondary to age-related macular degeneration imaged by optical coherence tomography angiography. Retina 2015; 35(11): 2212-2218.

23 Coscas G, Lupidi M, Coscas F, Francais C, Cagini C, Souied EH. Optical coherence tomography angiography during follow-up: qualitative and quantitative analysis of mixed type I and II choroidal neovascularization after vascular endothelial growth factor trap therapy. Ophthalmic Res 2015; 54(2): 57-63.
24 Tan AC, Dansingani KK, Yannuzzi LA, Sarraf D, Freund KB. Type 3 neovascularization imaged with cross-sectional and en face optical coherence tomography angiography. Retina 2016; 37(2): 234-246.

25 Kuehlewein L, Dansingani KK, de Carlo TE, Bonini Filho MA, Iafe NA, Lenis TL et al. Optical coherence tomography angiography of type 3 neovascularization secondary to age-related macular degeneration. Retina 2015; 35(11): 2229-2235.

26 Tsai AS, Cheung N, Gan AT, Jaffe GJ, Sivaprasad S, Wong TY et al. Retinal angiomatous proliferation. Surv Ophthalmol 2017; 62(4): 462-46492.

27 Coscas GJ, Lupidi M, Coscas F, Cagini C, Souied EH. Optical coherence tomography angiography versus traditional multimodal imaging in assessing the activity of exudative age-related macular degeneration: a new diagnostic challenge. Retina 2015; 35(11): 2219-2228.

28 Marques JP, Costa JF, Marques M, Cachulo ML, Figueira J, Silva R. Sequential morphological changes in the CNV net after intravitreal anti-VEGF evaluated with OCT angiography. Ophthalmic Res 2016; 55(3): 145-151.

29 Muakkassa NW, Chin AT, de Carlo T, Klein KA, Baumal CR, Witkin AJ et al. Characterizing the effect of anti-vascular endothelial growth factor therapy on treatment-naive choroidal neovascularization using optical coherence tomography angiography. Retina 2015; 35(11): 2252-2259.

30 Spaide RF. Optical coherence tomography angiography signs of vascular abnormalization with antiangiogenic therapy for choroidal neovascularization. Am J Ophthalmol 2015; 160(1): 6-16.

31 Inoue M, Balaratnasingam C, Freund KB. Optical coherence tomography angiography of polypoidal choroidal vasculopathy and polypoidal choroidal neovascularization. Retina 2015; 35(11): 2265-2274.

32 Tomiyasu T, Nozaki M, Yoshida M, Ogura Y. Characteristics of polypoidal choroidal vasculopathy evaluated by optical coherence tomography angiography. Invest Ophthalmol Vis Sci 2016; 57(9): OCT324-OCT330.

33 Tanaka K, Mori R, Kawamura A, Nakashizuka H, Wakatsuki Y, Yuzawa M. Comparison of OCT angiography and indocyanine green angiographic findings with subtypes of polypoidal choroidal vasculopathy. $\mathrm{Br} \mathrm{J}$ Ophthalmol 2017; 101(1): 51-55.

34 Cheung CM, Yanagi Y, Mohla A, Lee SY, Mathur R, Chan CM et al. Characterization and differentiation of polypoidal choroidal vasculopathy using swept source optical coherence tomography angiography. Retina 2016; 37(8): 1464-1474

35 Wang M, Zhou Y, Gao SS, Liu W, Huang Y, Huang D et al. Evaluating polypoidal choroidal vasculopathy with optical coherence tomography angiography. Invest Ophthalmol Vis Sci 2016; 57(9): OCT526-OCT532.

36 Srour M, Querques G, Semoun O, El Ameen A, Miere A, Sikorav A et al. Optical coherence tomography angiography characteristics of polypoidal choroidal vasculopathy. $\mathrm{Br} \mathrm{J}$ Opthalmol 2016; 100(11): 1489-1493.

37 Takayama K, Ito Y, Kaneko H, Kataoka K, Sugita T, Maruko $\mathrm{R}$ et al. Comparison of indocyanine green angiography and optical coherence tomographic angiography in polypoidal choroidal vasculopathy. Eye 2017; 31(1): 45-52.

38 Miere A, Semoun O, Cohen SY, El Ameen A, Srour M, Jung $C$ et al. Optical coherence tomography angiography 
features of subretinal fibrosis in age-related macular degeneration. Retina 2015; 35(11): 2275-2284.

39 Dansingani KK, Balaratnasingam C, Klufas MA, Sarraf D, Freund KB. Optical coherence tomography angiography of shallow irregular pigment epithelial detachments in pachychoroid spectrum disease. Am J Ophthalmol 2015.

40 Dansingani KK, Balaratnasingam C, Klufas MA, Sarraf D, Freund KB. Optical coherence tomography angiography of shallow irregular pigment epithelial detachments in pachychoroid spectrum disease. Am J Ophthalmol 2015; 160(6): 1243-1254. e1242.

41 Carnevali A, Cicinelli MV, Capuano V, Corvi F, Mazzaferro A, Querques L et al. Optical coherence tomography angiography: a useful tool for diagnosis of treatment-naive quiescent choroidal neovascularization. Am J Ophthalmol 2016; 169: 189-198.

42 Roisman L, Zhang Q, Wang RK, Gregori G, Zhang A, Chen CL et al. Optical coherence tomography angiography of asymptomatic neovascularization in intermediate age-related macular degeneration. Ophthalmology 2016; 123(6): 1309-1319.

43 Palejwala NV, Jia Y, Gao SS, Flaxel CJ, Hwang TS, Lauer AK et al. Detection of nonexudative choroidal neovascularization in age-related macular degeneration with optical coherence tomography angiography. Retina 2015; 35(11): 2204-2211.

44 Waheed NK, Moult EM, Fujimoto JG, Rosenfeld PJ. Optical coherence tomography angiography of dry age-related macular degeneration. Dev Ophthalmol 2016; 56: 91-100.

45 Moult EM, Waheed NK, Novais EA, Choi W, Lee B, Ploner SB et al. Swept-source optical coherence tomography angiography reveals choriocapillaris alterations in eyes with nascent geographic atrophy and drusen-associated geographic atrophy. Retina 2016; 36(Suppl 1): S2-S11.

46 Toto L, Borrelli E, Di Antonio L, Carpineto P, Mastropasqua R. Retinal vascular plexuses' changes in dry age-related macular degeneration, evaluated by means of optical coherence tomography angiography. Retina 2016; 36(8): 1566-1572.

47 Al-Sheikh M, Phasukkijwatana N, Dolz-Marco R, Rahimi M, Iafe NA, Freund KB et al. Quantitative OCT angiography of the retinal microvasculature and the choriocapillaris in myopic eyes. Invest Ophthalmol VIs Sci 2017; 58(4): 2063-2069.

48 Querques L, Giuffre C, Corvi F, Zucchiatti I, Carnevali A, De Vitis LA et al. Optical coherence tomography angiography of myopic choroidal neovascularisation. Br J Ophthalmol 2017; 101(5): 609-615.

49 Liu B, Bao L, Zhang J. Optical coherence tomography angiography of pathological myopia sourced and idiopathic choroidal neovascularization with follow-up. Medicine 2016; 95(14): e3264.

50 Maruko I, Spaide RF, Koizumi H, Sawaguchi S, Izumi T, Hasegawa $\mathrm{T}$ et al. Choroidal blood flow visualization in high myopia using a projection artifact method in optical coherence tomography angiography. Retina 2017; 37(3): 460-465.

51 Dimitrova G, Chihara E, Takahashi H, Amano H, Okazaki K. Quantitative retinal optical coherence tomography angiography in patients with diabetes without diabetic retinopathy. Invest Ophthalmol Vis Sci 2017; 58(1): 190-196.

52 Soares M, Neves C, Marques IP, Pires I, Schwartz C, Costa MÂ et al. Comparison of diabetic retinopathy classification using fluorescein angiography and optical coherence tomography angiography. Br J Ophthalmol 2017; 101(1): 62-68.

53 Krawitz BD, Mo S, Geyman LS, Agemy SA, Scripsema NK, Garcia PM et al. Acircularity index and axis ratio of the foveal avascular zone in diabetic eyes and healthy controls measured by optical coherence tomography angiography. Vis Res; e-pub ahead of print 26 February 2017; doi: 10.1016/j.visres.2016.09.019.

54 Ghasemi Falavarjani K, Iafe NA, Hubschman JP, Tsui I, Sadda SR, Sarraf D. Optical coherence tomography angiography analysis of the foveal avascular zone and macular vessel density after anti-vegf therapy in eyes with diabetic macular edema and retinal vein occlusion. Invest Ophthalmol Vis Sci 2017; 58(1): 30-34.

55 Ishibazawa A, Nagaoka T, Takahashi A, Omae T, Tani T, Sogawa $\mathrm{K}$ et al. Optical coherence tomography angiography in diabetic retinopathy: a prospective pilot study. Am J Ophthalmol 2015; 160(1): 35-44 e31.

56 Salz DA, de Carlo TE, Adhi M, Moult E, Choi W, Baumal CR et al. Select features of diabetic retinopathy on swept-source optical coherence tomographic angiography compared with fluorescein angiography and normal eyes. JAMA Ophthalmol 2016; 134(6): 644-650.

57 de Carlo TE, Chin AT, Bonini Filho MA, Adhi M, Branchini L, Salz DA et al. Detection of microvascular changes in eyes of patients with diabetes but not clinical diabetic retinopathy using optical coherence tomography angiography. Retina 2015; 35(11): 2364-2370.

58 Takase N, Nozaki M, Kato A, Ozeki H, Yoshida M, Ogura Y. Enlargement of foveal avascular zone in diabetic eyes evaluated by en face optical coherence tomography angiography. Retina 2015; 35(11): 2377-2383.

59 Samara WA, Shahlaee A, Adam MK, Khan MA, Chiang A, Maguire JI et al. Quantification of diabetic macular ischemia using optical coherence tomography angiography and its relationship with visual acuity. Ophthalmology 2017; 124(2): 235-244.

60 Ishibazawa A, Nagaoka T, Yokota H, Takahashi A, Omae T, Song YS et al. Characteristics of retinal neovascularization in proliferative diabetic retinopathy imaged by optical coherence tomography angiography. Invest Ophthalmol Vis Sci 2016; 57(14): 6247-6255.

61 Singh A, Agarwal A, Mahajan S, Karkhur S, Singh R, Bansal R et al. Morphological differences between optic disc collaterals and neovascularization on optical coherence tomography angiography. Graefes Arch Clin Exp Ophthalmol 2016; 255(4): 753-759.

62 Hwang TS, Jia Y, Gao SS, Bailey ST, Lauer AK, Flaxel CJ et al. Optical coherence tomography angiography features of diabetic retinopathy. Retina 2015; 35(11): 2371-2376.

63 Agemy SA, Scripsema NK, Shah CM, Chui T, Garcia PM, Lee JG et al. Retinal vascular perfusion density mapping using optical coherence tomography angiography in normals and diabetic retinopathy patients. Retina 2015; 35(11): 2353-2363.

64 Kim AY, Chu Z, Shahidzadeh A, Wang RK, Puliafito CA, Kashani AH. Quantifying microvascular density and morphology in diabetic retinopathy using spectral-domain optical coherence tomography angiography. Invest Ophthalmol Vis Sci 2016; 57(9): OCT362-OCT370.

65 Zhang M, Hwang TS, Dongye C, Wilson DJ, Huang D, Jia Y. Automated quantification of nonperfusion in three retinal plexuses using projection-resolved optical coherence 
tomography angiography in diabetic retinopathy. Invest Ophthalmol Vis Sci 2016; 57(13): 5101-5106.

66 Ting DS, Tan GS, Agrawal R, Yanagi Y, Sie NM, Wong CW et al. Optical coherence tomographic angiography in type 2 diabetes and diabetic retinopathy. JAMA Ophthalmol 2017; 135(4): 306-312.

67 Iafe NA, Phasukkijwatana N, Chen X, Sarraf D. Retinal capillary density and foveal avascular zone area are age-dependent: quantitative analysis using optical coherence tomography angiography. Invest Ophthalmol Vis Sci 2016; 57(13): 5780-5787.

68 Bonini Filho MA, Adhi M, de Carlo TE, Ferrara D, Baumal CR, Witkin AJ et al. Optical coherence tomography angiography in retinal artery occlusion. Retina 2015; 35(11): 2339-2346.

69 Kashani AH, Lee SY, Moshfeghi A, Durbin MK, Puliafito CA. Optical coherence tomography angiography of retinal venous occlusion. Retina 2015; 35(11): 2323-2331.

70 Kadomoto S, Muraoka Y, Ooto S, Miwa Y, Iida Y, Suzuma $\mathrm{K}$ et al. Evaluation of macular ischemia in eyes with branch retinal vein occlusion: an optical coherence tomography angiography study. Retina; e-pub ahead of print 17 February 2017; doi: 10.1097/ IAE.0000000000001541.

71 Nobre Cardoso J, Keane PA, Sim DA, Bradley P, Agrawal R, Addison PK et al. Systematic evaluation of optical coherence tomography angiography in retinal vein occlusion. Am J Ophthalmol 2016; 163(93-107): e106.

72 Coscas F, Glacet-Bernard A, Miere A, Caillaux V, Uzzan J, Lupidi $\mathrm{M}$ et al. Optical coherence tomography angiography in retinal vein occlusion: evaluation of superficial and deep capillary plexa. Am J Ophthalmol 2016; 161(160-171): e161-e162.

73 Suzuki N, Hirano Y, Yoshida M, Tomiyasu T, Uemura A, Yasukawa $\mathrm{T}$ et al. Microvascular abnormalities on optical coherence tomography angiography in macular edema associated with branch retinal vein occlusion. Am J Ophthalmol 2016; 161(126-132): e121.

74 de Castro-Abeger AH, de Carlo TE, Duker JS, Baumal CR. Optical coherence tomography angiography compared to fluorescein angiography in branch retinal artery occlusion. Ophthalmic Surg Lasers Imaging Retina 2015; 46(10): 1052-1054.

75 Kang JW, Yoo R, Jo YH, Kim HC. Correlation of microvascular structures on optical coherence tomography angiography with visual acuity in retinal vein occlusion. Retina 2017; 37(9): 1700-1709.

76 Casselholmde Salles M, Kvanta A, Amren U, Epstein D. Optical coherence tomography angiography in central retinal vein occlusion: correlation between the foveal avascular zone and visual acuity. Invest Ophthalmol Vis Sci 2016; 57(9): OCT242-OCT246.

77 Sellam A, Glacet-Bernard A, Coscas F, Miere A, Coscas G, Souied EH. Qualitative and quantitative follow-up using optical coherence tomography angiography of retinal vein occlusion treated with anti-VEGF: optical coherence tomography angiography follow-up of retinal vein occlusion. Retina 2016; 37(6): 1176-1184.

78 Matet A, Daruich A, Dirani A, Ambresin A, Behar-Cohen F. Macular telangiectasia type 1: capillary density and microvascular abnormalities assessed by optical coherence tomography angiography. Am J Ophthalmol. 2016; 167: $18-30$.
79 Spaide RF, Klancnik Jr JM, Cooney MJ, Yannuzzi LA, Balaratnasingam C, Dansingani KK et al. Volume-rendering optical coherence tomography angiography of macular telangiectasia type 2. Ophthalmology 2015; 122(11): 2261-2269.

80 Spaide RF, Suzuki M, Yannuzzi LA, Matet A, Behar-Cohen F. Volume-rendered angiographic and structural optical coherence tomography angiography of macular telangiectasia type 2. Retina 2017; 37(3): 424-435.

81 Chidambara L, Gadde SG, Yadav NK, Jayadev C, Bhanushali D, Appaji AM et al. Characteristics and quantification of vascular changes in macular telangiectasia type 2 on optical coherence tomography angiography. Br J Ophthalmol 2016; 100(11): 1482-1488.

82 Toto L, Di Antonio L, Mastropasqua R, Mattei PA, Carpineto P, Borrelli E et al. Multimodal imaging of macular telangiectasia type 2: focus on vascular changes using optical coherence tomography angiography. Invest Ophthalmol Vis Sci 2016; 57(9): OCT268-OCT276.

83 Denniston AK, Dick AD. Systemic therapies for inflammatory eye disease: past, present and future. $B M C$ Ophthalmol 2013; 13: 18.

84 Kim AY, Rodger DC, Shahidzadeh A, Chu Z, Koulisis N, Burkemper B et al. Quantifying retinal microvascular changes in uveitis using spectral-domain optical coherence tomography angiography. Am J Ophthalmol 2016; 171: 101-112.

85 Levison AL, Baynes KM, Lowder CY, Kaiser PK, Srivastava SK. Choroidal neovascularisation on optical coherence tomography angiography in punctate inner choroidopathy and multifocal choroiditis. $\mathrm{Br} J$ Ophthalmol 2016; 101(5): 616-622.

86 Zahid S, Chen KC, Jung JJ, Balaratnasingam C, Ghadiali Q, Sorenson $\mathrm{J}$ et al. Optical coherence tomography angiography of chorioretinal lesions due to idiopathic multifocal choroiditis. Retina 2016; 37(8): 1451-1463.

87 Cheng L, Chen X, Weng S, Mao L, Gong Y, Yu S et al. Spectral-domain optical coherence tomography angiography findings in multifocal choroiditis with active lesions. Am J Ophthalmol 2016; 169: 145-161.

88 Chen KC, Marsiglia M, Dolz-Marco R, Zahid S, Mrejen S, Pulido JS et al. Foveal exudate and choroidal neovascularization in atypical cases of multiple evanescent white dot syndrome. Retina; e-pub ahead of print 16 January 2017; doi: 10.1097/IAE.0000000000001486.

89 de Carlo TE, Bonini Filho MA, Adhi M, Duker JS. Retinal and choroidal vasculature in birdshot chorioretinopathy analyzed using spectral domain optical coherence tomography angiography. Retina 2015; 35(11): 2392-2399.

90 Roberts PK, Nesper PL, Goldstein DA, Fawzi AA. Retinal capillary density in patients with birdshot

chorioretinopathy. Retina; e-pub ahead of print 10 February 2017; doi:10.1097/IAE.0000000000001543.

91 Khairallah M, Abroug N, Khochtali S, Jelliti B, Coscas G, Lupidi $\mathrm{M}$ et al. Optical coherence tomography angiography in patients with behcet uveitis. Retina 2017; 37(9): 1678-1691.

92 Aggarwal K, Agarwal A, Deokar A, Mahajan S, Singh R, Bansal R et al. Distinguishing features of acute Vogt-Koyanagi-Harada disease and acute central serous chorioretinopathy on optical coherence tomography angiography and en face optical coherence tomography imaging. J Ophthalmic Inflamm Infect 2017; 7(1): 3. 
93 Heiferman MJ, Rahmani S, Jampol LM, Nesper PL, Skondra D, Kim LA et al. Acute posterior multifocal placoid pigment epitheliopathy on optical coherence tomography angiography. Retina; e-pub ahead of print 1 February 2017; doi:10.1097/IAE.0000000000001487.

94 Pichi F, Srivastava SK, Levinson A, Baynes KM, Traut C, Lowder CY. A focal chorioretinal bartonella lesion analyzed by optical coherence tomography angiography. Ophthalmic Surg Lasers Imaging Retina 2016; 47(6): 585-588.

95 Jia Y, Wei E, Wang X, Zhang X, Morrison JC, Parikh M et al. Optical coherence tomography angiography of optic disc perfusion in glaucoma. Ophthalmology 2014; 121(7): 1322-1332.

96 Liu L, Jia Y, Takusagawa HL, Pechauer AD, Edmunds B, Lombardi L et al. Optical coherence tomography angiography of the peripapillary retina in glaucoma. JAMA Ophthalmol 2015; 133(9): 1045-1052.

97 Yarmohammadi A, Zangwill LM, Diniz-Filho A, Suh MH, Yousefi S, Saunders LJ et al. Relationship between optical coherence tomography angiography vessel density and severity of visual field loss in glaucoma. Ophthalmology 2016; 123(12): 2498-2508.

98 Akil H, Huang AS, Francis BA, Sadda SR, Chopra V. Retinal vessel density from optical coherence tomography angiography to differentiate early glaucoma, pre-perimetric glaucoma and normal eyes. PLoS One 2017; 12(2): e0170476.

99 Ang M, Sng C, Milea D. Optical coherence tomography angiography in dural carotid-cavernous sinus fistula. $B M C$ Ophthalmol 2016; 16: 93.

100 Sharma S, Ang M, Najjar RP, Sng C, Cheung CY, Rukmini AV et al. Optical coherence tomography angiography in acute non-arteritic anterior ischaemic optic neuropathy. Br J Ophthalmol 2017; 101(8): 1045-1051.

101 Ghasemi Falavarjani K, Tian JJ, Akil H, Garcia GA, Sadda SR, Sadun AA. Swept-source optical coherence tomography angiography of the optic disk in optic neuropathy. Retina 2016; 36(Suppl 1): S168-S177.

102 Wang X, Jia Y, Spain R, Potsaid B, Liu JJ, Baumann B et al. Optical coherence tomography angiography of optic nerve head and parafovea in multiple sclerosis. $\mathrm{Br} J$ Ophthalmol 2014; 98(10): 1368-1373.

103 Higashiyama T, Nishida Y, Ohji M. Optical coherence tomography angiography in eyes with good visual acuity recovery after treatment for optic neuritis. PloS One 2017; 12(2): e0172168.

104 Ang M, Cai Y, Shahipasand S, Sim DA, Keane PA, Sng CC et al. En face optical coherence tomography angiography for corneal neovascularisation. Br J Ophthalmol 2015; 100(5): 616-621.

105 Ang M, Sim DA, Keane PA, Sng CC, Egan CA, Tufail A et al. Optical coherence tomography angiography for anterior segment vasculature imaging. Ophthalmology 2015; 122(9): 1740-1747.

106 Ang M, Cai Y, Tan AC. Swept source optical coherence tomography angiography for contact lens-related corneal vascularization. J Ophthalmol 2016; 2016: 9685297.

107 Ang M, Cai Y, MacPhee B, Sim DA, Keane PA, Sng CC et al. Optical coherence tomography angiography and indocyanine green angiography for corneal vascularisation. Br J Ophthalmol 2016; 100(11): 1557-1563.

108 Ang M, Cai Y, Shahipasand S, Sim DA, Keane PA, Sng CC et al. En face optical coherence tomography angiography for corneal neovascularisation. Br J Ophthalmol. 2016; 100(5): 616-621.

109 Ploner SB, Moult EM, Choi W, Waheed NK, Lee B, Novais EA et al. Toward quantitative optical coherence tomography angiography: visualizing blood flow speeds in ocular pathology using variable interscan time analysis. Retina 2016; 36(Suppl 1): S118-S126. 